\title{
Eugenol-Preconditioned Mesenchymal Stem Cell-Derived Extracellular Vesicles Promote Antioxidant Capacity of Tendon Stem Cells In Vitro and In Vivo
}

\author{
Xiangze Li, ${ }^{1}$ Zhan Su, ${ }^{2}$ Kaiying Shen,, ${ }^{1}$ Q Wang, ${ }^{1}$ Chencheng Xu, ${ }^{1}$ Fuqiang Wang, ${ }^{3}$ \\ Yuchi Zhang, ${ }^{3}$ and Dapeng Jiang $\mathbb{D}^{1}$ \\ ${ }^{1}$ Department of General Surgery, Shanghai Children's Medical Center, Shanghai Jiao Tong University School of Medicine, \\ Shanghai, China \\ ${ }^{2}$ Department of Orthopaedics, Heilongjiang Red Cross Sengong General Hospital, Heilongjiang, China \\ ${ }^{3}$ Department of Pediatric Surgery, Hongqi Hospital, Mudanjiang Medical University, Mudanjiang, China
}

Correspondence should be addressed to Dapeng Jiang; jdp509@163.com

Received 5 November 2021; Revised 6 January 2022; Accepted 12 January 2022; Published 8 February 2022

Academic Editor: Tullia Maraldi

Copyright (c) 2022 Xiangze Li et al. This is an open access article distributed under the Creative Commons Attribution License, which permits unrestricted use, distribution, and reproduction in any medium, provided the original work is properly cited.

\begin{abstract}
Tendon stem cells (TSCs) are often exposed to oxidative stress at tendon injury sites, which impairs their physiological effect as well as therapeutic application. Recently, extracellular vesicles (EVs) derived from bone marrow mesenchymal stem cells (BMSCs) were shown to mediate cell protection and survival under stress conditions. The function of BMSC-EVs may be affected by pretreatment with various factors such as eugenol (EUG) - a powerful antioxidant. In our previous study, we found that $\mathrm{H}_{2} \mathrm{O}_{2}$ significantly impaired TSC proliferation and tenogenic differentiation capabilities. Apoptosis and intracellular ROS accumulation in TSCs were induced by $\mathrm{H}_{2} \mathrm{O}_{2}$. However, such $\mathrm{H}_{2} \mathrm{O}_{2}$-induced damage was prevented by treatment with EUGBMSC-EVs. Furthermore, EUG-BMSC-EVs activated the Nrf2/HO-1 pathway to counteract $\mathrm{H}_{2} \mathrm{O}_{2}$-induced damage in TSCs. In a rat patellar tendon injury model, the ROS level was significantly higher than that in the normal tendon and TSCs not pretreated showed a poor therapeutic effect. However, EUG-BMSC-EV-pretreated TSCs significantly improved tenogenesis and matrix regeneration during tendon healing. Additionally, the EUG-BMSC-EV group had a significantly improved fiber arrangement. Overall, EUG-BMSC-EVs protected TSCs against oxidative stress and enhanced their functions in tendon injury. These findings provide a basis for potential clinical use of EUG-BMSC-EVs as a new therapeutic vehicle to facilitate TSC therapies for tendon regeneration.
\end{abstract}

\section{Introduction}

Tendons play important roles in transmitting forces from muscles to the skeleton. They are frequently injured by mechanical loading during both occupational and gymnastic activities [1]. In clinical medicine, improving the healing effectiveness after tendon injury remains a major challenge. The healing process of tendons is slow because of the inability of tendons to self-repair and the poor regenerative capability of tenocytes [2]. In recent years, tenocyte- and mesenchymal stromal cell-based therapeutic strategies have been applied to tendon injuries [3-5]. However, tenocytes and mesenchymal stromal cells may not be ideal cell sources for tendon repair because of their limited proliferative capability and the risk of ectopic bone formation [6]. Recently, tendon stem cells (TSCs) were shown to have potential for the regeneration of injured tendons [7-9]. However, nontenocyte differentiation and TSC dysfunction may occur after their transplantation because the local biochemical environment undergoes complicated changes after injuries, such as inflammation and oxidative stress $[10,11]$.

Previous studies have shown that oxidative stress diminishes the abilities of TSCs to self-renew, proliferate, and differentiate into tenocytes $[12,13]$. TSC damage caused by oxidative stress is a major contributor to tendon degeneration and tendinopathy. It has also been reported that 
antagonizing posttraumatic oxidative stress with vitamin $\mathrm{C}$ reduces tendon adhesion [14]. Moreover, the proliferation and expression of tendon-related markers in TSCs are suppressed significantly by $\mathrm{H}_{2} \mathrm{O}_{2}$ treatment [10]. Consequently, it is necessary to find regulators that improve the function of TSCs under oxidative stress.

Extracellular vesicles released from bone marrow mesenchymal stem cells (BMSC-EVs) have recently been shown to mediate tissue regeneration as well as cell protection and survival under various pathophysiological conditions [15-17]. Recent studies have also demonstrated that MSCEVs positively modulate the function of stem cells $[18,19]$. However, BMSC-EVs have been found to play an antiinflammatory role in tendon injury while their antioxidant capacity needs to be improved. As a consequence, BMSCEV treatment does not achieve the goal of complete healing $[20]$.

In recent years, cell preconditioning has attracted increasing attention. Preconditioning of cells with cytokines, hypoxic conditions, and small molecule compounds increases their capabilities [21-23]. Exosomes derived from pretreated MSCs also have therapeutic effects [24]. Eugenol (EUG) is a natural compound extracted from vegetable oil. It has a wide range of pharmacological effects that include antioxidant, anti-inflammatory, antibacterial, and antiviral effects [25]. Previous studies have demonstrated that EUGpretreated adipogenic MSCs have enhanced antifibrosis abilities [26]. On the basis of the antioxidant effect of EUG, we speculated that preconditioning of BMSCs with EUG may improve the antioxidant effect of BMSC-EVs.

We hypothesized that EUG-BMSC-EVs may have a novel role in protecting TSCs against oxidative stressinduced damage. To test this hypothesis, EVs were isolated from BMSCs preconditioned with EUG. Then, the effects of EUG-BMSC-EVs on proliferation, apoptotic activity, tenocyte phenotype, and reactive oxygen species (ROS) accumulation in TSCs were investigated in vitro. The effects of TSCs pretreated with EUG-BMSC-EVs on mending tendon tissues were also characterized using a rat patellar tendon injury model.

\section{Methods}

2.1. Animals. Animal experiments were approved by the Animal Care and Use Committee, Shanghai Children's Medical Center, Shanghai Jiao Tong University School of Medicine (China). Male Sprague Dawley (SD) rats weighing $180-220 \mathrm{~g}$ at 8-10 weeks of age were provided by the Shanghai SLAC Laboratory Animal Company (No: SCMCDWFLL-20200026).

2.2. Isolation and Culture of Rat BMSCs and TSCs. BMSCs were isolated from the bone marrow of SD rats as described previously [27]. Briefly, bone marrow was extracted from the femurs and tibias of rats and cultured in Dulbecco's modified Eagle's medium (Cat: C11885500BT, Gibco, USA) containing 10\% fetal bovine serum (Cat: 10099141C, Gibco, USA) and $1 \%$ penicillin/streptomycin (Cat: 15140122,
Gibco, USA). The medium was changed every 2 days. The BMSCs used in this study were between passages 3 and 5 .

The isolation and culture methods of TSCs were previously established [28]. For primary cell cultures, patellar tendons were removed and cut into small sections; then, the fragments were digested with dispase (Cat: D4693, SigmaAldrich, USA) and type I collagenase (Cat: C0130, SigmaAldrich, USA) at $37^{\circ} \mathrm{C}$ for $1 \mathrm{~h}$. The isolated cells were cultured in plates in Dulbecco's modified Eagle's medium containing $10 \%$ fetal bovine serum and $1 \%$ penicillin/ streptomycin. TSCs formed colonies on the culture plates after 8-10 days. Cells from P2 to P3 after isolation were used in all experiments.

2.3. Preconditioning of BMSCs. EUG (Cat: E51791, SigmaAldrich, USA) was dissolved in ethanol. BMSCs were seeded in 96-well plates at a density of 5000 cells per well. Cells were rinsed with PBS after $24 \mathrm{~h}$ and preconditioned with EUG for another $24 \mathrm{~h}$. After that, CCK8 assay was used to evaluate cell viability. $50 \mu \mathrm{M}$ EUG was chosen for our experiment.

For preconditioning, BMSCs were cultured in a complete medium until $70 \%$ confluent; then, they were washed twice with PBS. Serum-free medium was added for $24 \mathrm{~h}$, followed by EUG-containing complete medium for $24 \mathrm{~h}$. The BMSC group was added ethanol-containing complete medium.

2.4. Isolation and Identification of EVs. After preconditioning for $24 \mathrm{~h}$, nonpreconditioned and EUG-preconditioned BMSCs were rinsed with PBS and cultured in a medium containing $10 \%$ exosome-depleted fetal bovine serum at $37^{\circ} \mathrm{C}$ for an additional $48 \mathrm{~h}$. The culture medium was collected for obtaining EVs. The culture medium was centrifugated at $300 \times \mathrm{g}$ for $10 \mathrm{~min}$ and $2000 \times \mathrm{g}$ for $10 \mathrm{~min}$ to remove cellular debris. Then, the supernatant was centrifuged at $10,000 \times \mathrm{g}$ for $30 \mathrm{~min}$. After that, cell-free supernatants were ultracentrifuged at $100,000 \times \mathrm{g}$ for $70 \mathrm{~min}$ twice. $50 \mu \mathrm{L}$ PBS was used to resuspend the EVs, and the resulting solution was used for downstream experiments. The total protein content in EVs was detected using a BCA protein assay kit (Cat: P0010, Beyotime, China). Western blotting, nanoparticle tracking analysis (NTA), and transmission electron microscopy (TEM) were used to identify the collected EVs. We also extracted protein from BMSCs for comparison.

2.5. Internalization of DiI PKH26-Labeled EVs into TSCs. BMSC-EVs and EUG-BMSC-EVs were labeled with DiI PKH26 (Cat: MINI26, Sigma-Aldrich, USA) as previously described [24]. EVs were washed in PBS to wash unbound DiI away. Then, TSCs were incubated with DiI-labeled EVs $(10 \mu \mathrm{g})$ for $12 \mathrm{~h}$. TSCs were then fixed in $4 \%$ paraformaldehyde after being washed in PBS for three times and incubated with Hoechst 33342 (Cat: C1026, Beyotime, China) for $5 \mathrm{~min}$ at room temperature. Stained cells were observed under a laser confocal microscope.

2.6. $\mathrm{H}_{2} \mathrm{O}_{2}$ and EV Treatments. TSCs were subjected to $0.5 \mathrm{mM} \mathrm{H}_{2} \mathrm{O}_{2}$ for up to $24 \mathrm{~h}$ as described previously [29]. As soon as cells were exposed to $\mathrm{H}_{2} \mathrm{O}_{2}$, they were incubated in either control medium or medium containing BMSC-EVs 
or EUG-BMSC-EVs. To investigate whether the protective effect of EVs was associated with the activation of Nrf2/ HO-1, TSCs were pretreated with ML385 (an Nrf2 inhibitor, Cat: HY-100523, Sigma-Aldrich, USA) for $4 \mathrm{~h}$ and cotreated with $\mathrm{H}_{2} \mathrm{O}_{2}$ for $24 \mathrm{~h}$.

2.7. Cell Viability Assay. For cell viability assays, TSCs were plated in 96-well culture plates at a density of $5 \times 10^{3}$ cells/ well. After $24 \mathrm{~h}$ in culture, cells were treated with EVs and $\mathrm{H}_{2} \mathrm{O}_{2}$. Next, TSCs were cultured for another $24 \mathrm{~h}$ prior to assessing proliferation. The viability of TSCs was determined using the CCK8 assay.

2.8. Colony-Forming Assay. TSCs were seeded in culture dishes at 500 cells/well and incubated in DMEM supplemented with EVs and $\mathrm{H}_{2} \mathrm{O}_{2}$ for a further 7 days. The cells were stained with $0.5 \%$ Crystal Violet Staining Solution (Cat: C0121, Beyotime, China) for $10 \mathrm{~min}$ for counting the number of cell colonies. Colonies of less than $2 \mathrm{~mm}$ in diameter were ignored under a microscope.

2.9. Apoptosis Assay. TSC apoptosis was determined by annexin V-fluorescein isothiocyanate (FITC)/propidium iodide (PI) double staining. Harvested cells were suspended in 1x binding buffer (Cat: C1069M, Beyotime, China) and stained with FITC-conjugated annexin V and PI. Cells were analyzed by flow cytometry (FACSCanto ${ }^{\mathrm{TM}}$, BD, USA) within $1 \mathrm{~h}$.

2.10. Detection of Intracellular ROS Accumulation. To assess intracellular peroxide accumulation, cells were stained with DCFH-DA (Cat: S0033M, Beyotime, China). Cells were analyzed under a fluorescence microscope. Fluorescence intensity was calculated for the quantitative analysis of ROS accumulation.

2.11. Animal Experiments. Generation of the rat patellar tendon injury model and the surgical procedures were performed as in previous studies [20]. The central one-third of the patellar tendon was removed to create a tendon injury. 12 rats were randomly divided into 2 groups (6 rats/group). In group II, the central one-third of the patellar tendon was removed to create a tendon injury and then we injected $30 \mu \mathrm{L}$ of fibrin sealant. However, rats in group I only got a skin incision at the same site. At 2 days after surgery, rats were sacrificed. The normal tendon in group I and the injured tendon in group II was collected and processed via cryosectioning for further analysis. DCFH-DA was used to detect ROS produced in a tendon.

64 rats underwent surgery for partial resection of the patellar tendon followed by treatment immediately. Rats were randomly divided into 4 groups (16 rats/group). In the control group, the defect was filled with $30 \mu \mathrm{L}$ volume of fibrin sealant alone. In the TSC group, $30 \mu \mathrm{L}$ volume of fibrin with $5 \times 10^{4}$ TSCs (without BMSC-EV treatment) was injected into the tendon injury. In the BMSC-EV+TSC group, $30 \mu \mathrm{L}$ volume of fibrin with $5 \times 10^{4}$ TSCs (pretreated with $60 \mu \mathrm{g} / \mathrm{mL}$ BMSC-EVs for $24 \mathrm{~h}$ ) was inoculated into the tendon injury. In the EUG-BMSC-EV+TSC group, $30 \mu \mathrm{L}$ volume of fibrin with $5 \times 10^{4}$ TSCs (pretreated with $60 \mu \mathrm{g}$ /
mL EUG-BMSC-EVs for $24 \mathrm{~h}$ ) was inoculated into the tendon injury. At 1 and 2 weeks after surgery, 8 rats in each group were sacrificed, and the injured patellar tendons were harvested for histology, immunohistochemistry, protein, and mRNA analysis.

2.12. Histology and Immunohistochemistry. At weeks 1 and 2 after treatments, collected rat patellar tendon tissues were fixed in 4\% paraformaldehyde (Cat: G1101, Servicebio, China) for $24 \mathrm{~h}$. Specimens were used for immunohistochemistry to examine the expression of proliferating cell nuclear antigen (PCNA, Cat: 10205-2-AP, dilution: 1:500, Proteintech, USA), tenascin C (TNC, Cat: 67710-1-Ig, dilution: $1: 500$, Proteintech, USA), tenomodulin (TNMD, Cat: ab203676, dilution: 1:400, Abcam, UK), scleraxis (SCXA, Cat: DF13293, dilution: $1: 400$, Affinity, USA), collagen type I (COLI, Cat: 66761-1-Ig, dilution: 1:500, Proteintech, USA), and collagen type III (COLIII, Cat: 22734-1-AP, dilution: $1: 500$, Proteintech, USA). The specimens were embedded in paraffin. Sections of $4 \mu \mathrm{m}$ thickness were cut and deparaffinized in xylene followed by hydration, and then, they were placed in 3\% hydrogen peroxide to block endogenous activity. Blocking of the sections was performed with $5 \%$ bovine serum albumin for $20 \mathrm{~min}$. After that, sections were incubated overnight at $4^{\circ} \mathrm{C}$ with primary antibodies. Slides were then incubated with a secondary antibody in the dark at $37^{\circ} \mathrm{C}$ for $30 \mathrm{~min}$. Images were observed with a digital pathology slide scanner (KF-PRO-120, KFBIO).

The specimens were also used for hematoxylin-eosin (H\&E) or Masson trichrome staining. Histopathological analysis of the stained rat patellar tendon tissue was performed using the fiber alignment score as previously described: $0=0 \%$ to $25 \%$ parallel fiber alignment; $1=25 \%$ to $50 \%$ parallel fiber alignment; $2=50 \%$ to $75 \%$ parallel fiber alignment; and $3=75 \%$ to $100 \%$ parallel fiber alignment [14].

2.13. Reverse Transcription-Quantitative Polymerase Chain Reaction (RT-qPCR). Gene expression in cells and healing tendons was determined via real-time PCR. Total RNA was extracted with Trizol reagent (Cat: 15596018, Invitrogen, USA). cDNA was synthesized using a PrimeScript ${ }^{\mathrm{TM}}$ RT Master Mix Kit (Cat: RR036A, TAKARA, China). RTPCR was carried out with the CFX96 Real-Time PCR Detection System (Bio-Rad, USA). Total RNA isolation, cDNA synthesis, and gene expression assays were performed as in previous studies [29]. Glyceraldehyde-3-phosphate dehydrogenase (GAPDH) was used as an endogenous reference gene. Relative gene expression levels were analyzed with the $2^{\triangle \Delta C T}$ formula and then normalized to controls.

Rat-specific primers used for TNMD, SCX, TNC, Cat, PCNA, Sod1, Nfe212, HO-1, collagen type I, collagen type III, bFGF, and GAPDH are shown in Table 1.

2.14. Western Blotting Analysis. RIPA lysis buffer (Cat: C1053, Applygen, China) containing Cocktail (50x) (Cat: P1265, Applygen, China) was used to prepare tissues and cells. After isolating proteins from homogenates, immunoblotting was performed overnight at $4^{\circ} \mathrm{C}$ using the following 
TABLe 1: Rat-specific primers used for qRT-PCR analysis.

\begin{tabular}{|c|c|c|}
\hline \multirow{2}{*}{ TNMD } & Forward & $5^{\prime}$-CCAGACAAGCAAGCGAGGAAGAC-3' \\
\hline & Reverse & $5^{\prime}$-ACAGACCCTGCGGCAGTAGC-3' \\
\hline \multirow{2}{*}{ SCX } & Forward & $5^{\prime}$-CAACGTGCTACTGGTGGGTGAAG-3' \\
\hline & Reverse & 5'-TGTTCTCGCCGCCGTCTCTG-3' \\
\hline \multirow{2}{*}{ TNC } & Forward & 5'-AAAGCAGCCACCCGCTATTA-3' \\
\hline & Reverse & 5' -TCAGGTTCTTTGGCTGTGGAG-3' \\
\hline \multirow{2}{*}{ PCNA } & Forward & $5^{\prime}$-CGGCGTGAACCTACAGAGCATG-3' \\
\hline & Reverse & $5^{\prime}$-GCAGCGGTATGTGTCGAAGCC-3' \\
\hline \multirow{2}{*}{ Sod1 } & Forward & 5' -TGGCGGTCCAGCGGATGAAG-3' \\
\hline & Reverse & $5^{\prime}$-CGGCCAATGATGGAATGCTCTCC-3' \\
\hline \multirow{2}{*}{ Cat } & Forward & $5^{\prime}$-GCGAATGGAGAGGCAGTGTACTG-3' \\
\hline & Reverse & 5'-GGTCTTCCTGTGCAAGTCTTCCTG-3' \\
\hline \multirow{2}{*}{$\mathrm{Nfe} 2 \mathrm{l} 2$} & Forward & 5'-GCCTTCCTCTGCTGCCATTAGTC-3' \\
\hline & Reverse & 5'-TGCCTTCAGTGTGCTTCTGGTTG-3' \\
\hline \multirow{2}{*}{$\mathrm{HO}-1$} & Forward & $5^{\prime}$-CAGACAGAGTTTCTTCGCCAGAGG-3' \\
\hline & Reverse & 5'-TGTGAGGACCCATCGCAGGAG-3' \\
\hline \multirow{2}{*}{ Colla1 } & Forward & 5'-AGAAAGGATCTCCTGGTGC-3' \\
\hline & Reverse & 5'-ACGTTCACCACTTGCTCCA-3' \\
\hline \multirow{2}{*}{ Col3a1 } & Forward & $5^{\prime}$-TGCACCTGGCAAAAACGG-3' \\
\hline & Reverse & $5^{\prime}$-TTCCATTTTCTCCTGGAGG-3' \\
\hline \multirow{2}{*}{ bFGF } & Forward & $5^{\prime}$-GACGATGACGATGATGATGACTCCTC-3' \\
\hline & Reverse & $5^{\prime}$-GTAACGAACCTTGTAGCCTCCGATC-3' \\
\hline \multirow{2}{*}{ GAPDH } & Forward & $5^{\prime}$-TGACTCTACCCACGGCAAGTTCAA-3' \\
\hline & Reverse & $5^{\prime}$-ACGACATACTCAGCACCAGCATCA-3' \\
\hline
\end{tabular}

rabbit primary antibodies: Col III (Cat: 22734-1-AP, dilution: 1:1000, Proteintech, USA), Col I (Cat: 66761-1-Ig, dilution: 1:1000, Proteintech, USA), CD9 (Cat: ab263019, dilution: $1: 1000$,Abcam, UK), CD63 (Cat: ab134045, dilution: 1:1000, Abcam, UK), TSG101 (Cat: ab133586, dilution: $1: 1000$, Abcam, UK), TNC (Cat: 67710-1-Ig, dilution: $1: 1000$, Proteintech, USA), TNMD (Cat: ab203676, dilution: 1:1000, Abcam, UK), SCXA (Cat: DF13293, dilution: $1: 1000$, Affinity, USA), PCNA (Cat: 10205-2-AP, dilution: $1: 1000$, Proteintech, USA), poly ADP-ribose polymerase 1 (PARP1, Cat: 13371-1-AP, dilution: 1:1000, Proteintech, USA), catalase (Cat: 21260-1-AP, dilution: $1: 1000$, Proteintech, USA), and GAPDH (Cat: 60004-1-Ig, dilution: 1: 1000, Proteintech, USA). Horseradish peroxidase- (HRP-) conjugated secondary antibodies (Cat: 15015\&15014, dilution: $1: 10000$, Proteintech, China) were then incubated with the membranes for $1 \mathrm{~h}$ at room temperature. Chemiluminescent signals were developed with an enhanced chemiluminescence (Millipore, USA) and detected by the ChemiDoc imaging system (Tanon, China).

2.15. Statistical Analysis. Experiments were carried out more than three times in vitro and in vivo. All data were expressed as means \pm standard deviation from at least three separate experiments. We used Student's $t$-test to compare differences between two groups. In order to compare differences in more than two groups, analysis of variance followed by Tukey's multiple comparison test was used. $P<0.05$ was considered statistically significant. The analysis was all performed in GraphPad Prism (GraphPad Software Inc., USA).

\section{Results}

3.1. Characterization and Internalization of BMSC-EVs. BMSCs were positive for cell surface markers, such as CD90 (99.88\%) and CD44 (62.00\%), but negative for the hematopoietic markers CD34 (3.04\%) and C11b (0.29\%) (Figure 1(a)). We also analyzed the morphology and multipotency of BMSCs (Figures 1(b) and 1(c)). We characterized BMSC-EVs and EUG-BMSC-EVs through TEM, NTA analysis, and western blotting. TEM analysis showed that the shape of EVs was round or spherical (Figures 1(d) and $1(\mathrm{e})$ ). Western blotting analysis revealed that the expression levels of CD9, HSP70, and TSG101 were significantly higher in EVs compared with BMSCs (Figure 1(f)). Moreover, as shown by nanoparticle tracking analysis (NTA), EVs were 

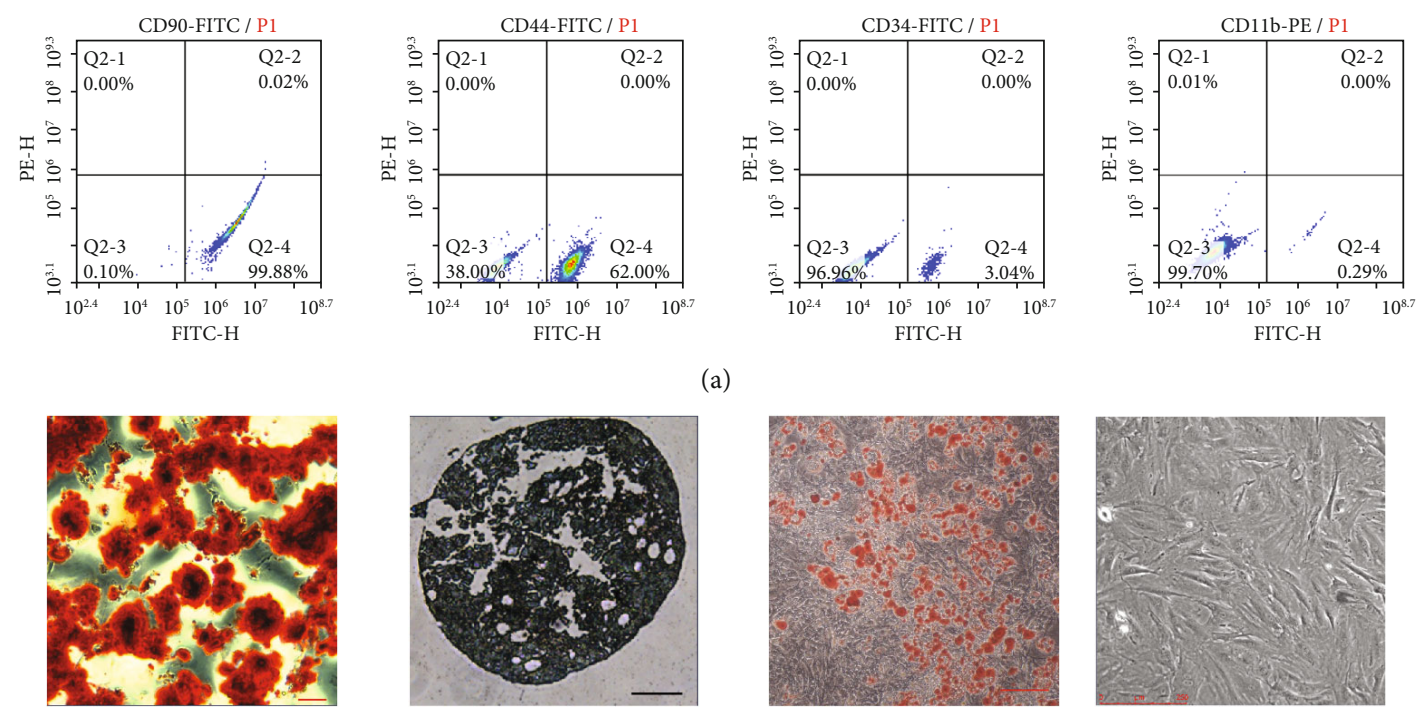

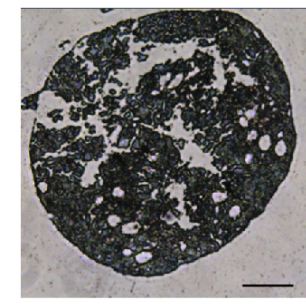

Chondrogenesis

(b)

BMSC-EVs

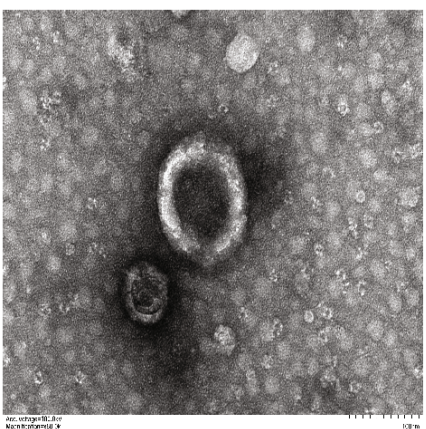

(d)

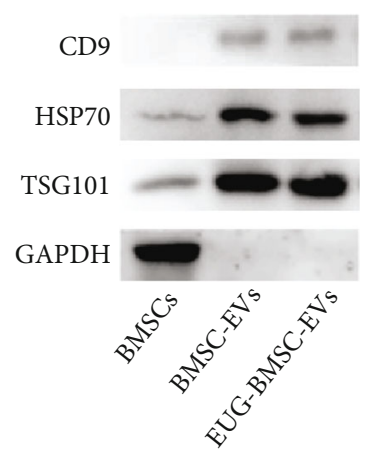

(f)

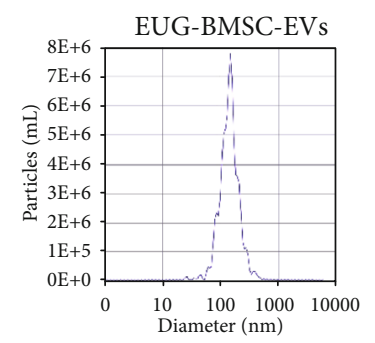

(h)

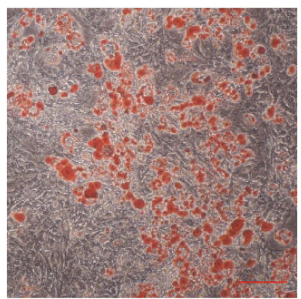

Adipogenesis

EUG-BMSC-EVs

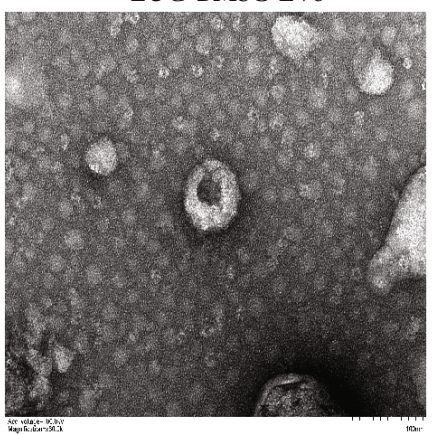

(e)

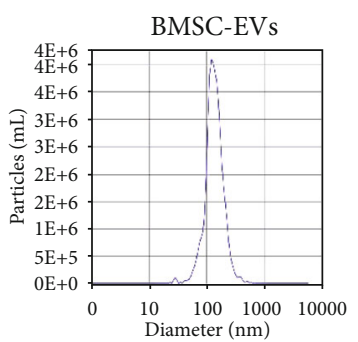

(g)

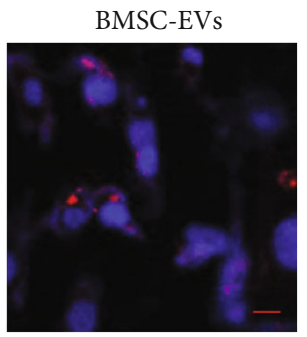

(i)

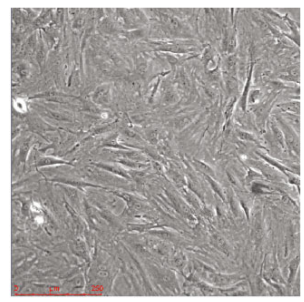

Morphology

(c)

Figure 1: Continued. 


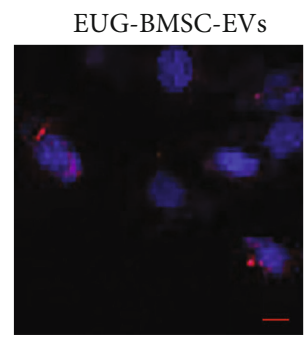

(j)

FIGURE 1: Characterization of BMSCs and EVs. (a) FACS analysis for detection of BMSC surface markers. (b) The osteogenic, chondrogenic, and adipogenic differentiation potentials of BMSCs. Scale bar: $200 \mu \mathrm{m}$. (c) Morphology of BMSCs. Scale bar: $250 \mu \mathrm{m}$. (d, e) Morphology using transmission electron microscopy. (f) Western blot was used to detect the markers of EVs. (g, h) NTA was used to examine the particle size distribution. (i, j) Internalization of EVs by TSCs. EVs were labeled with DiI PKH26 while the nuclei of TSCs were labeled with Hoechst 33342. Scale bar: $20 \mu \mathrm{m}$.

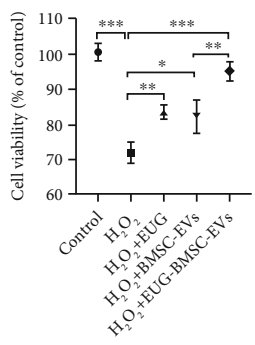

(a)

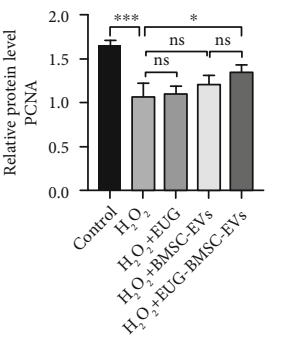

(c)

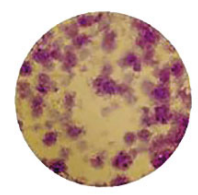

Control

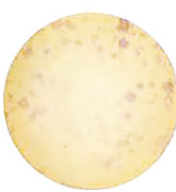

$\mathrm{H}_{2} \mathrm{O}_{2}$

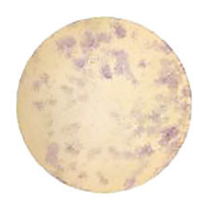

$\mathrm{H}_{2} \mathrm{O}_{2}+\mathrm{EUG}$

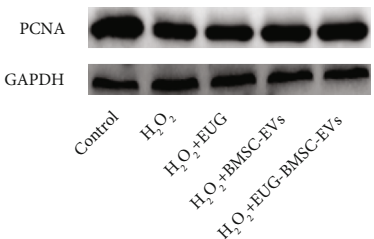

(b)

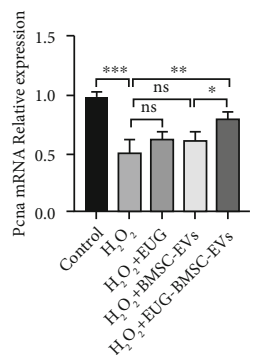

(d)

(e)

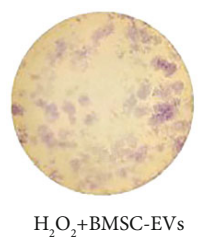

$\mathrm{H}_{2} \mathrm{O}_{2}+\mathrm{BMSC}-\mathrm{EVs}$

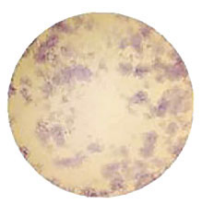

$\mathrm{H}_{2} \mathrm{O}_{2}+\mathrm{EUG}-\mathrm{BMSC}-\mathrm{EV} s$

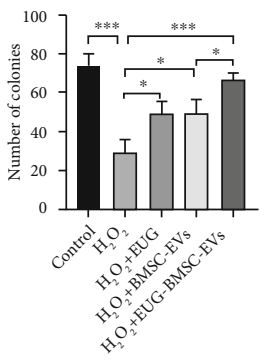

(f)

FIGURE 2: EUG-BMSC-EVs enhanced the viability and proliferation of $\mathrm{H}_{2} \mathrm{O}_{2}$-treated TSCs. (a) CCK8 assay was performed to assess TSC viability. (b, c) The protein and mRNA expression of PCNA in TSCs. (d) The expression of PCNA gene. (e, f) Colony-forming assay was performed to assess proliferation capacity of TSCs. ${ }^{*} P<0.05,{ }^{* *} P<0.01$, and ${ }^{* * *} P<0.001$.

uniform in size with a diameter of approximately $30-200 \mathrm{~nm}$; the average diameter of EVs was $130 \mathrm{~nm}$ (Figures 1(g) and $1(\mathrm{~h})$ ).

The internalization of EVs was observed under a laser confocal microscope. The red fluorescence of EVs was local- ized in the cytoplasm of TSCs, indicating that EVs were internalized by TSCs (Figures 1(i) and $1(\mathrm{j})$ ).

3.2. EUG-BMSC-EVs Enhanced the Viability and Proliferation of $\mathrm{H}_{2} \mathrm{O}_{2}$-Treated TSCs. We first examined the 


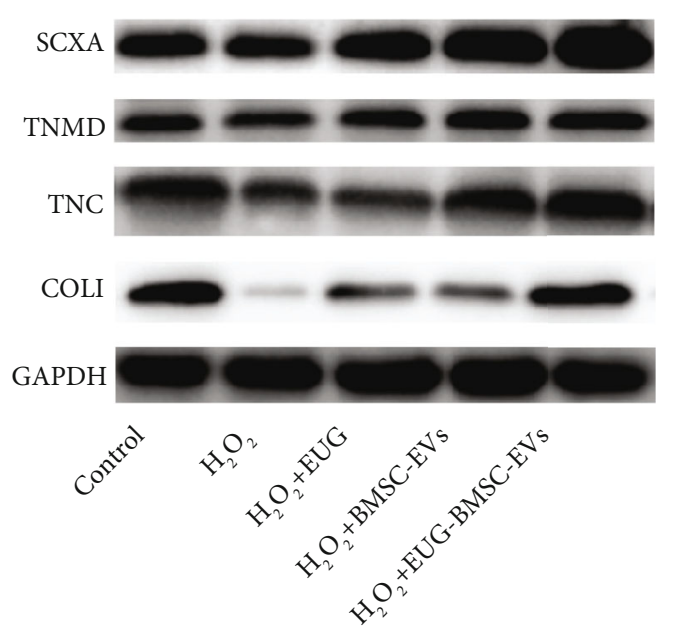

(a)

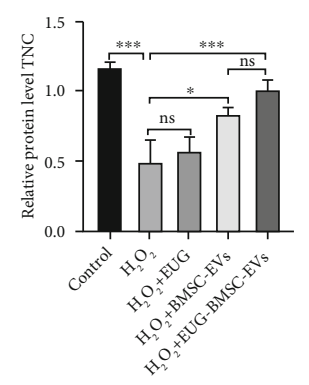

(d)

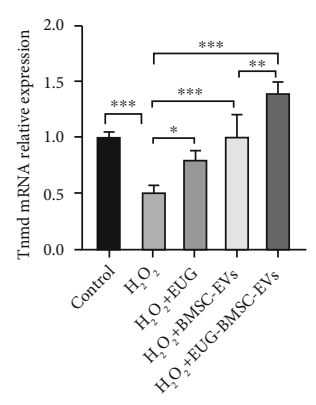

(g)

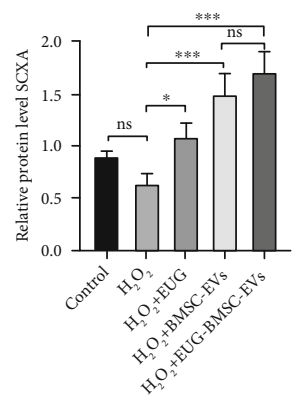

(b)

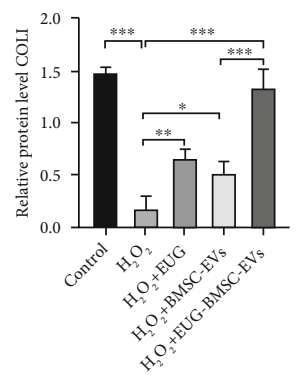

(e)

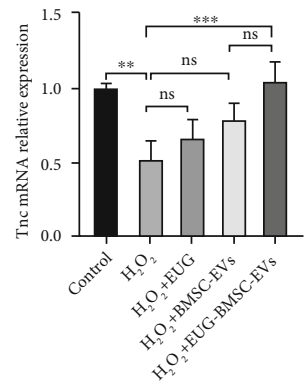

(h)

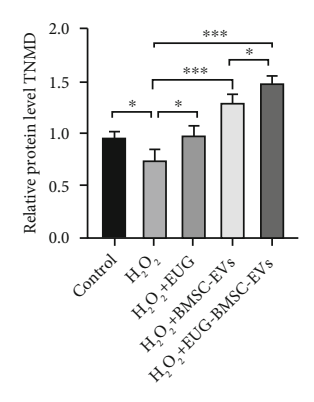

(c)

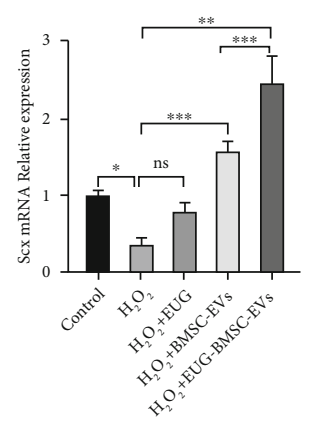

(f)

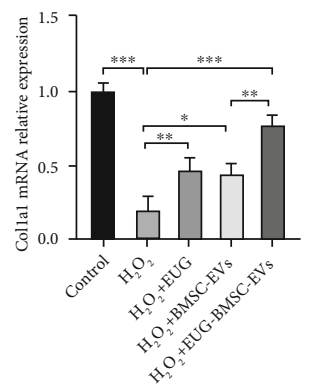

(i)

FIGURE 3: EUG-BMSC-EVs protected tenogenic differentiation of $\mathrm{H}_{2} \mathrm{O}_{2}$-treated TSCs. (a-e) Western blot was used to examine the expression of SCXA, TNMD, TNC, and COLI. (f-i) The expression of tenocyte-related genes, SCX, TNMD, TNC, and Colla1. ${ }^{*} P<0.05$, ${ }^{* *} P<0.01$, and ${ }^{* * *} P<0.001$.

effects of $\mathrm{H}_{2} \mathrm{O}_{2}$ treatment on the viability of TSCs. As shown in Figure $2(\mathrm{a}), \mathrm{H}_{2} \mathrm{O}_{2}$ at a concentration of $0.5 \mathrm{mM}$ for $24 \mathrm{~h}$ significantly impaired the viability of TSCs compared with controls. TSCs exhibited enhanced viability after treatment with BMSC-EVs, and the EUG-BMSC-EVs group showed better viability.

$\mathrm{H}_{2} \mathrm{O}_{2}$ at a concentration of $0.5 \mathrm{mM}$ for $24 \mathrm{~h}$ significantly impaired the expression of PCNA and the colony formation capacity of TSCs. Nonpreconditioned BMSC-EVs can hardly increase the PCNA level. In contrast, EUG-BMSCEVs significantly increased the PCNA expression and enhanced the relative number and average size of colonies (Figures 2(b)-2(f)).
3.3. Effect of EUG-BMSC-EVs on the Tenogenic Differentiation of $\mathrm{H}_{2} \mathrm{O}_{2}$-Treated TSCs. We examined the level of COLI, TNC (a glycoprotein of the extracellular matrix), TNMD (tenogenesis-related marker), and SCXA (tenogenic transcription factor), which are related to tenogenic differentiation [30]. Their expression levels were decreased by $\mathrm{H}_{2} \mathrm{O}_{2}$ treatment, but EUG-BMSC-EVs reversed these effects (Figures 3(a)-3(i)). TSCs subjected to EUG-BMSC-EVs exhibited a significant increase in the expression of these markers compared with the group treated with $\mathrm{H}_{2} \mathrm{O}_{2}$. Although BMSC-EVs can also protect TSCs against $\mathrm{H}_{2} \mathrm{O}_{2}$, their effect was inferior to EUGBMSC-EVs. 

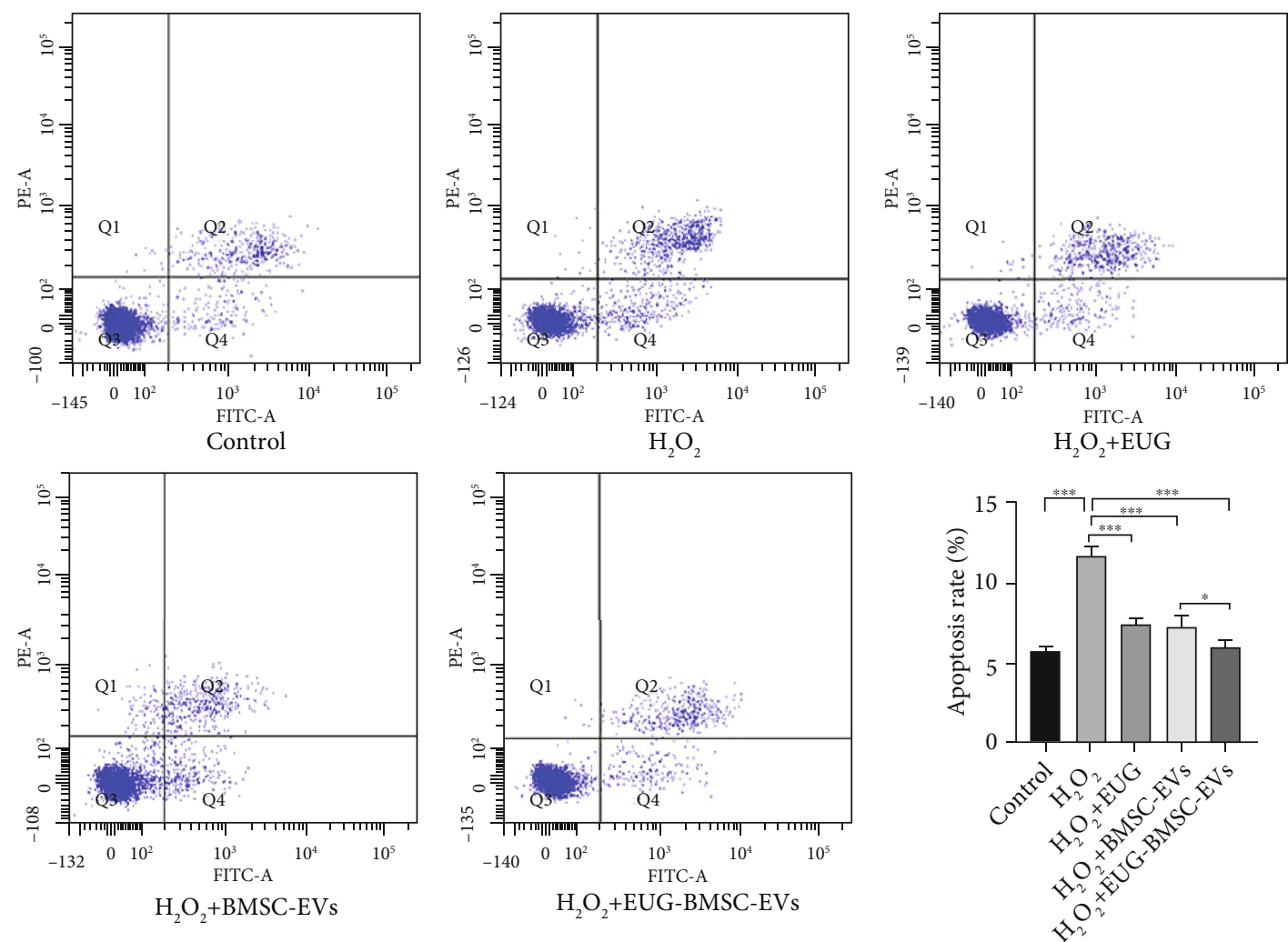

(a)

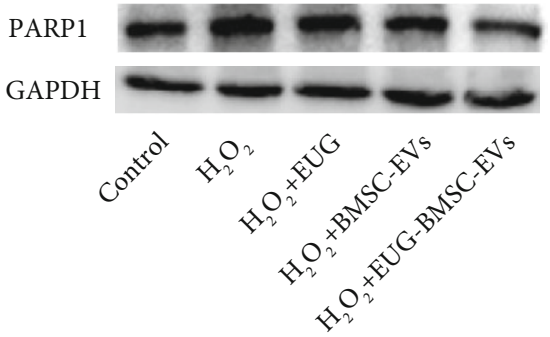

(b)

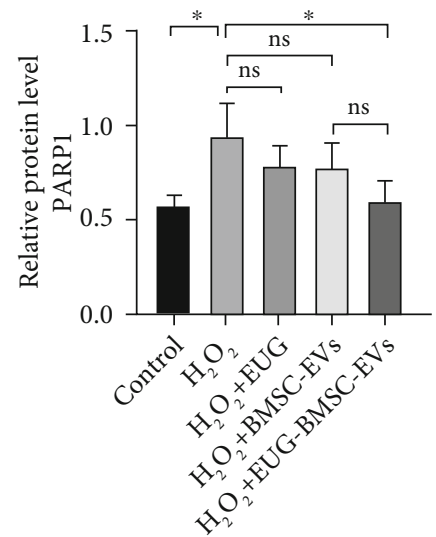

(c)

Figure 4: EUG-BMSC-EVs decreased $\mathrm{H}_{2} \mathrm{O}_{2}$-induced apoptosis. (a) Flow cytometry was used to assess the apoptosis of the TSCs. (b, c) Western blot was used to examine the expression of PARP1. ${ }^{*} P<0.05$ and ${ }^{* * *} P<0.001$.

3.4. Effect of EUG-BMSC-EVs on $\mathrm{H}_{2} \mathrm{O}_{2}$-Induced TSC Apoptosis. Apoptosis rates were gauged by flow cytometry analysis, which showed that $\mathrm{H}_{2} \mathrm{O}_{2}$ induced the apoptosis of TSCs after culture for $24 \mathrm{~h}$ in vitro. The proportion of apoptotic cells was $12 \% \pm 1 \%$ after treatment with $\mathrm{H}_{2} \mathrm{O}_{2}$. We next investigated the effects of EUG-BMSC-EVs on $\mathrm{H}_{2} \mathrm{O}_{2}$-induced apoptosis in TSCs. Treatment of TSCs with EUG and EVs decreased the percentage of apoptotic cells and the expression of PARP1, and EUG-BMSC-EVs were more effective than BMSC-EVs or EUG alone (Figures 4(a)-4(c)).
3.5. Effect of EUG-BMSC-EVs on Antioxidant Capacity of $\mathrm{H}_{2} \mathrm{O}_{2}$-Treated TSCs. Exposure to $\mathrm{H}_{2} \mathrm{O}_{2}$ leads to increased intracellular ROS generation. To examine the mechanisms underlying the protective effects of EUG-BMSC-EVs on TSCs, we also detected intracellular ROS level. The intensity of ROS fluorescence in cells was suppressed by treatment with EUG-BMSC-EVs (Figure 5(a)). We also detected the expression of SOD and catalase in $\mathrm{H}_{2} \mathrm{O}_{2}$-treated TSCs. As we predicted, EUG and BMSC-EVs increased the levels of catalase and SOD while both indexes were significantly higher in the EUG-BMSC-EVs group (Figures 5(b)-5(f)). 


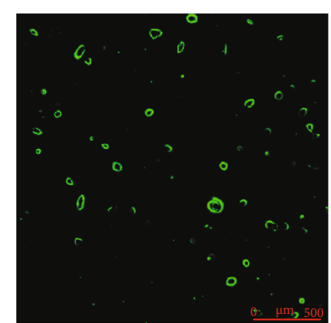

Control

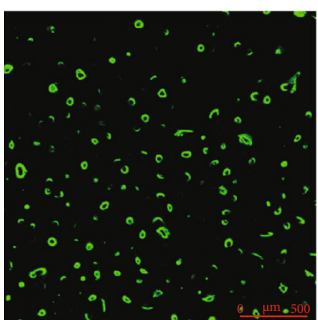

$\mathrm{H}_{2} \mathrm{O}_{2}+\mathrm{EUG}$

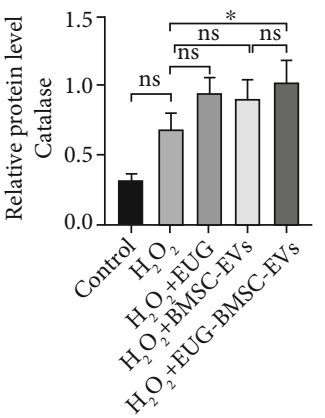

(c)

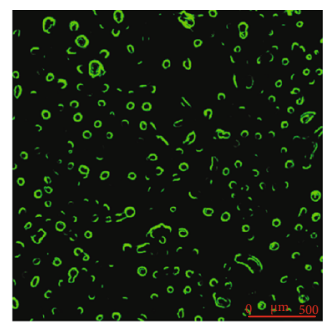

$\mathrm{H}_{2} \mathrm{O}_{2}$

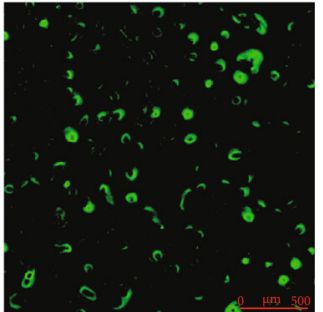

$\mathrm{H}_{2} \mathrm{O}_{2}+\mathrm{BMSC}-\mathrm{EVS}$

(a)

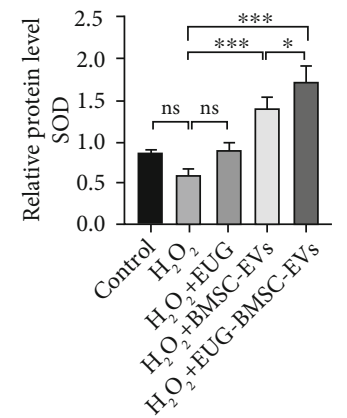

(d)
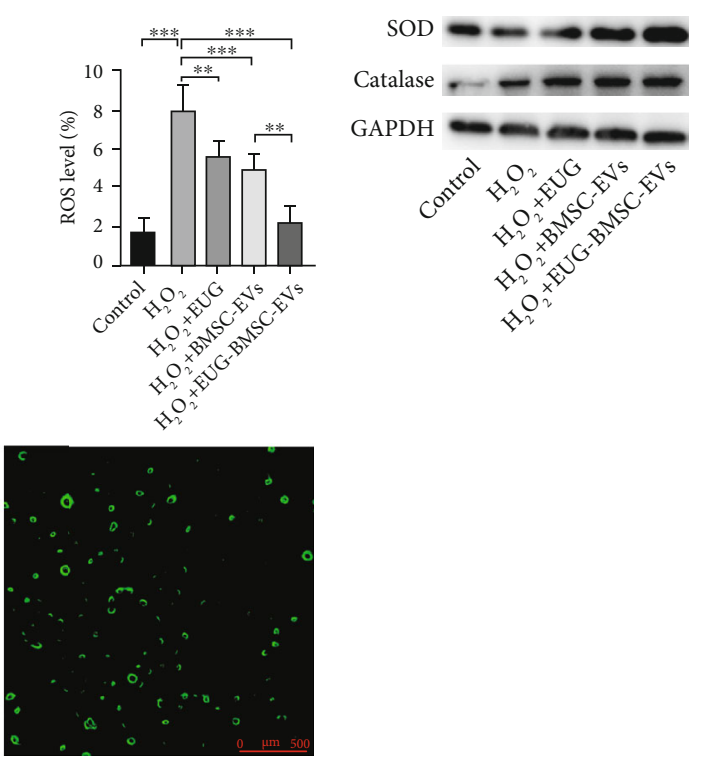

$\mathrm{H}_{2} \mathrm{O}_{2}+$ EUG-BMSC-EVs

(b)

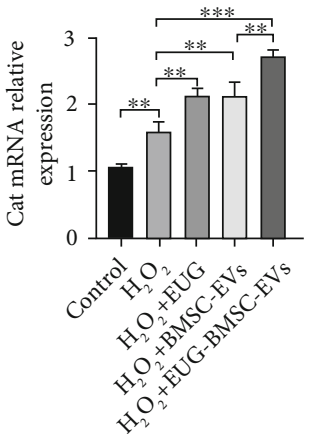

(e)

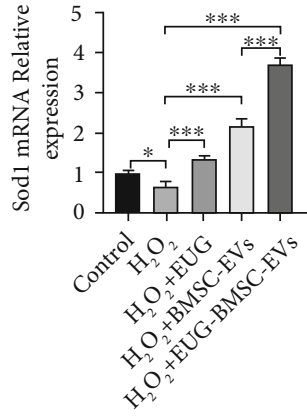

(f)

FIgURE 5: EUG-BMSC-EVs enhanced antioxidant capacity of $\mathrm{H}_{2} \mathrm{O}_{2}$-treated TSCs. (a) Detection of intracellular ROS accumulation by immunofluorescence assay. (b-d) Western blot was used to detect the expression of catalase and SOD. (e, f) The expression of Cat and Sod1 genes. Bars: $500 \mu \mathrm{m}$. Data are represented as mean $\pm \mathrm{SD}$. ${ }^{*} P<0.05,{ }^{* *} P<0.01$, and ${ }^{* * *} P<0.001$.

3.6. Nrf2/HO-1 Signaling Mediates the Protective Effects of EUG-BMSC-EVs on $\mathrm{H}_{2} \mathrm{O}_{2}$-Induced TSCs. Western blotting was performed to detect activation of the $\mathrm{Nrf} / \mathrm{HO}-1$ signaling pathway by EUG-BMSC-EVs in this study. EUG-BMSCEVs significantly augmented $\mathrm{Nrf} 2$ and $\mathrm{HO}-1$ expression levels in $\mathrm{H}_{2} \mathrm{O}_{2}$-induced TSCs compared with $\mathrm{H}_{2} \mathrm{O}_{2}$ only groups (Figures 6(a)-6(e)). To explore whether the cytoprotective effects of EUG-BMSC-EVs were dependent on Nrf2 activation, we pretreated TSCs with ML385 (an inhibitor of Nrf2). The result showed that ML385 dramatically attenuated the effect of EUG-BMSC-EVs on antiapoptosis and antioxidant in $\mathrm{H}_{2} \mathrm{O}_{2}$-treated TSCs. Moreover, inhibition of Nrf2 activation blocked the protective effects of EUGBMSC-EVs on the impaired cell proliferation and tenogenesis of $\mathrm{H}_{2} \mathrm{O}_{2}$-induced TSCs (Figures 6(f)-6(m)).

3.7. Effect of EUG-BMSC-EVs Pretreated TSCs on Tendon Morphology. We examine the ROS levels in a normal and injured tendon 2 days after surgery. The expression of ROS in an injured tendon was significantly higher than the normal tendon (Figures 7(a) and 7(b)). This phenomenon indicated intense oxidative stress at tendon injury sites.

We investigated the effects of TSCs pretreated with EUG-BMSC-EVs on tendon regeneration by H\&E and Masson's trichrome staining. We used fiber alignment score to evaluate the tendon: $0=0 \%$ to $25 \%$ parallel fiber alignment; $1=25 \%$ to $50 \%$ parallel fiber alignment; $2=50 \%$ to $75 \%$ parallel fiber alignment; and $3=75 \%$ to $100 \%$ parallel fiber alignment. At 1 week after implantation, organized and compact collagen fibers were observed in the EUG-BMSCEV-pretreated TSCs implantation groups (Figures 7 (c) and $7(d)$ ). Compared with the control groups, the EUG-BMSCEV-treated group showed significantly improved fiber arrangement. The fiber alignment score was significantly higher for the group treated with BMSC-EV-TSC compared with the control group and the TSC group (Figure $7(\mathrm{e})$ ). As we predicted, the EUG-BMSC-EVs promoted TSC functions better when compared with the BMSC-EV group. After 2 


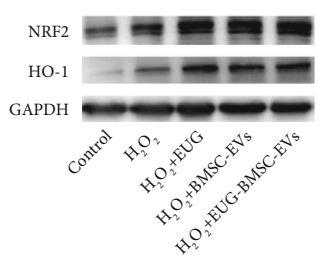

(a)

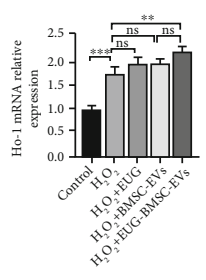

(e)
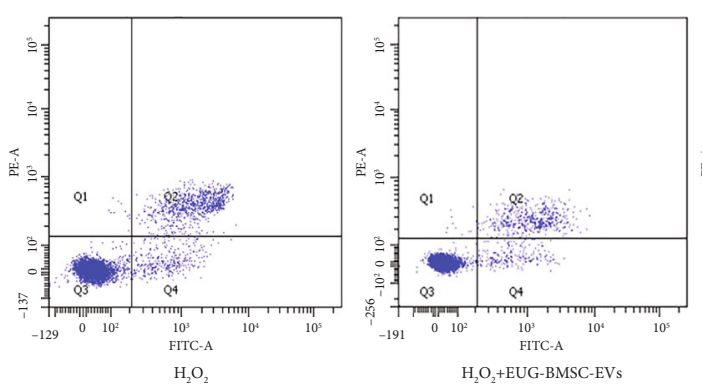

$\mathrm{H}_{2} \mathrm{O}_{2}+$ EUG-BMSC-EVs (f)

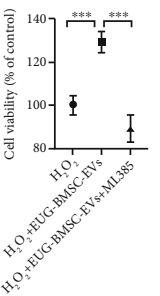

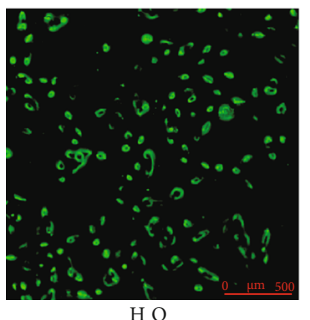

$\mathrm{H}_{2} \mathrm{O}_{2}$

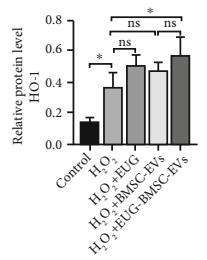

(c)

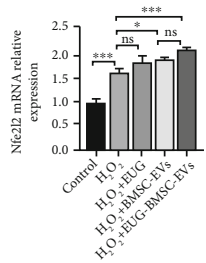

(d)

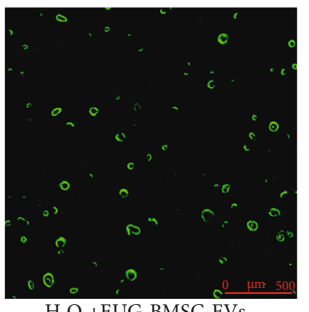

$\mathrm{H}_{2} \mathrm{O}_{2}+$ EUG-BMSC-EVs

(g)

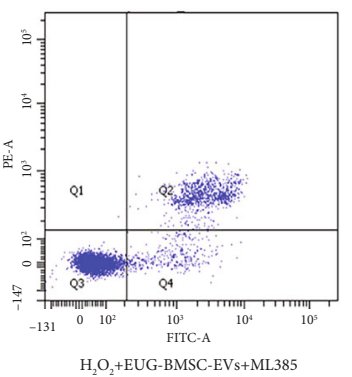

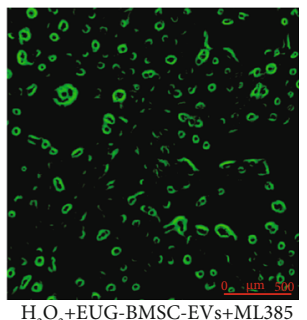

$\mathrm{H}_{2} \mathrm{O}_{2}+$ EUG-BMSC-EVs+ML385

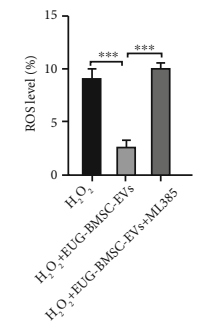

(h)
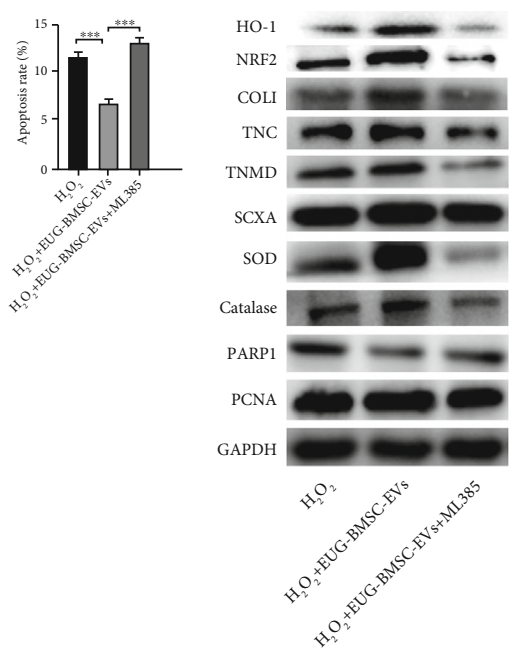

(k)

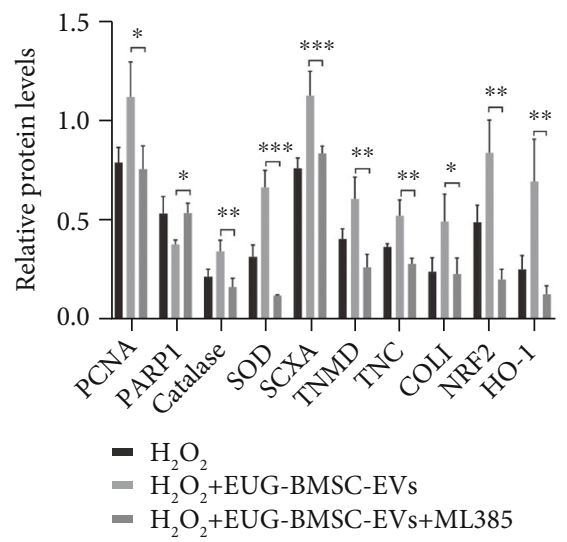

(l)

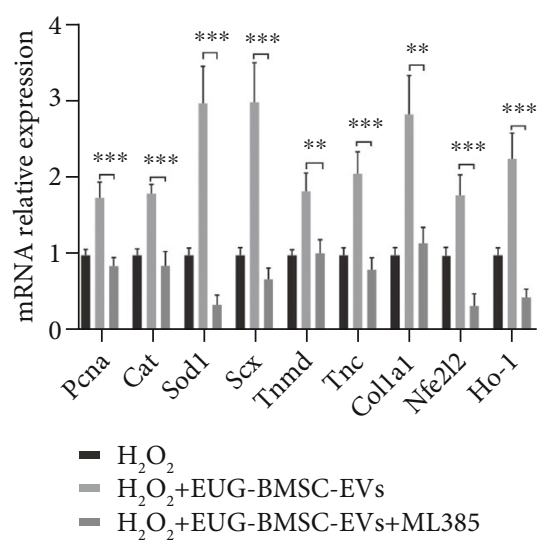

(m)

Figure 6: EUG-BMSC-EVs protected TSCs against $\mathrm{H}_{2} \mathrm{O}_{2}$ via $\mathrm{Nrf} 2 / \mathrm{HO}-1$ signaling pathway. (a-c) Western blot was used to examine the expression of NRF2 and HO-1 in $\mathrm{H}_{2} \mathrm{O}_{2}$-treated TSCs. (d, e) The expression of Nfe2l2 and HO-1 genes. (f) CCK8 assay was performed to assess TSC viability after ML385 treatment. ( $g, h$ ) Apoptosis of the TSCs after ML385 treatment was detected by flow cytometry. (i, $j$ ) The intracellular ROS accumulation was detected by immunofluorescence assay after ML385 treatment. (k, l) Western blot was used to examine the expression of PCNA, catalase, SOD, PARP1, SCXA, TNMD, TNC, COLI, NRF2, and HO-1 after ML385 treatment. (m) The expression of PCNA, Cat, Sod1, SCX, TNMD, TNC, Col1a1, Nfe212, and HO-1 genes after ML385 treatment. Bars: $500 \mu \mathrm{m}$. Data are represented as mean $\pm \mathrm{SD} .{ }^{*} P<0.05,{ }^{* *} P<0.01$, and ${ }^{* * *} P<0.001$. 


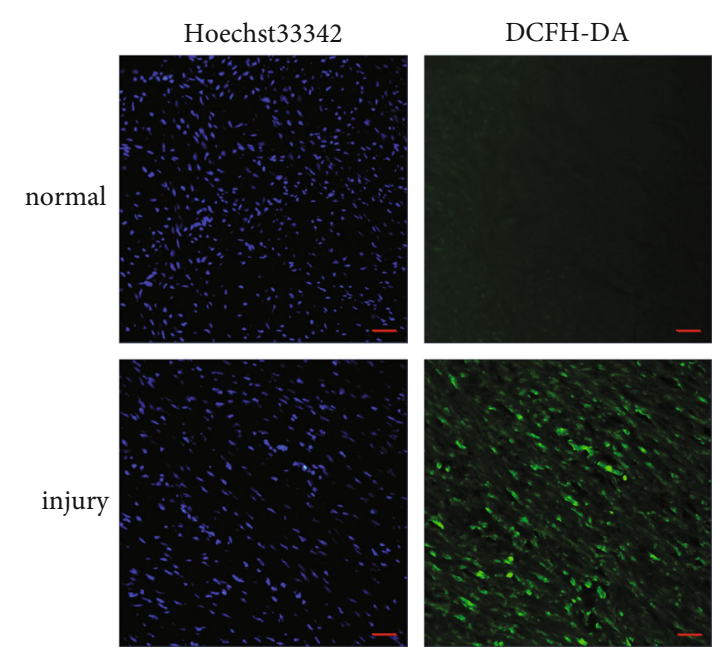

(a)
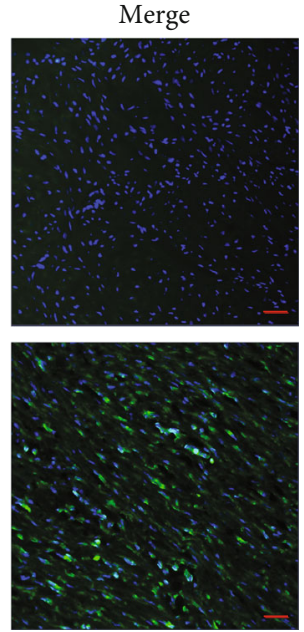

Week 1
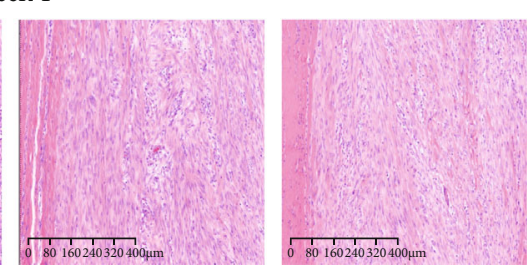

(c)
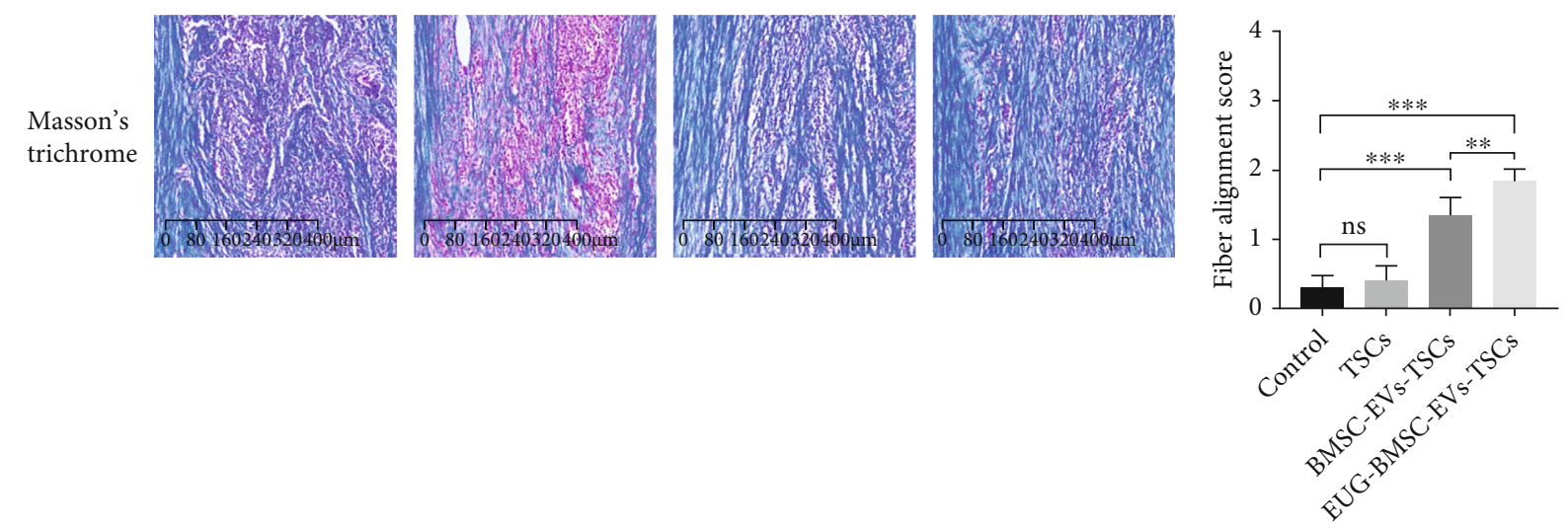

(d)

(e)

Week 2
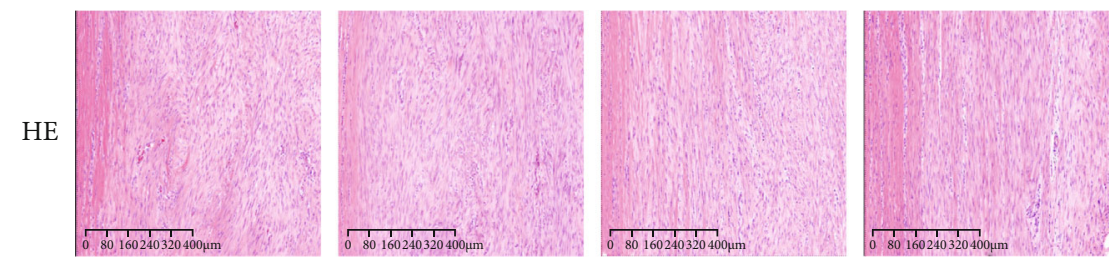

(f)

FIgURE 7: Continued. 


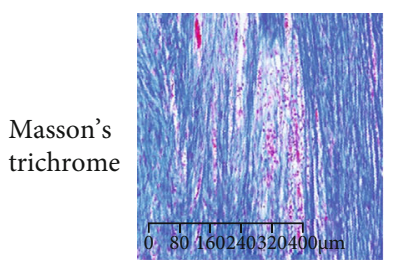

Control

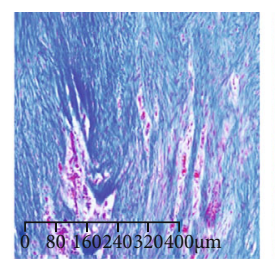

TSCs

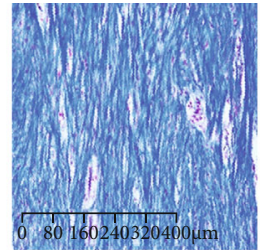

BMSC-EVs-TSCs

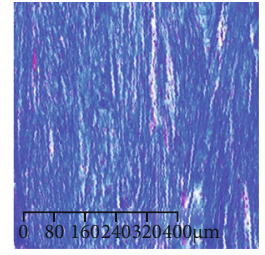

EUG-BMSC-EVs-TSCs

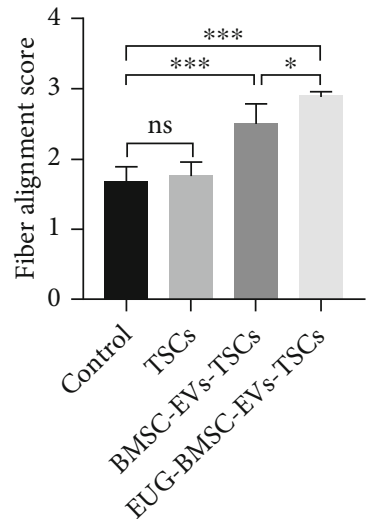

(h)

FIgURe 7: EUG-BMSC-EVs improved the healing of tendon injury. (a, b) ROS levels in normal and injured tendon. Bars: $200 \mu \mathrm{m}$. (c) The HE staining of tendon injury on the patellar tendon at 1 week. (d) Masson's trichrome staining of tendon injury at 1 week after surgery. (e) Fiber alignment score of the tendon injury rats $(n=8$ donors). (f) The HE staining of tendon injury on the patellar tendon at 2 weeks. (g) Masson's trichrome staining of tendon injury at 2 weeks after surgery. (h) Fiber alignment score of the tendon injury rats ( $n=8$ donors). Bars: $400 \mu \mathrm{m}$. Data are represented as mean $\pm \mathrm{SD} .{ }^{*} P<0.05,{ }^{* *} P<0.01$, and ${ }^{* * *} P<0.001$.

weeks, we analyze the fiber alignment score of each group. The results showed that the EUG-BMSC-EV-treated group almost achieved perfect healing (Figures $7(\mathrm{f})-7(\mathrm{~h})$ ).

\subsection{EUG-BMSC-EVs Enhanced Proliferation and} Tenogenesis of TSCs during Tendon Healing. The impact of TSCs on cell growth and differentiation during tendon healing was assessed in this study. At 1 week after injury, the expression of PCNA was higher in the EUG-BMSC-EV group. The expression of SCXA and TNMD was significantly elevated in the EUG-BMSC-EV-treated group compared with other groups. In addition, TNC was also enhanced in the EUG-BMSC-EV group. We also found that EVs derived from EUG-preconditioned BMSCs can significantly enhance TSC capability in tendon injury compared with BMSC-EVs. Basic fibroblast growth factor (bFGF) was reported to promote tendon repair. Compared with tendon tissues from other groups, repaired tendons from the TSC group (treated with EUG-BMSC-EVs) exhibited a higher level of bFGF (Figures 8(a) and 8(b)).

Immunohistochemical staining showed that PCNAexpressing cells were found in the center of the window defect at week 1. High expression of SCXA, TNMD, and TNC was observed in the EUG-BMSC-EV-treated group compared with other groups (Figure 8(c)). At 2 weeks after injury, the significant enhancement of PCNA, SCXA, TNMD, and bFGF had decreased while TNC remain higher in the EUG-BMSC-EV-treated group (Figures 9(a)-9(c)). We speculate that this phenomenon may correlate with better repair in the EUG-BMSC-EV group.

3.9. Effect of EUG-BMSC-EV-Pretreated TSCs on Matrix Regeneration during Tendon Healing. COLI was increased in healing tendons from the TSC group (treated with BMSC-EVs) compared with control tendons. The addition of BMSC-EV-treated TSCs to injured tendons also enhanced the COLIII expression level in repaired tendons (Figures 10(a) and 10(b)). When BMSC was preconditioned with EVs, EVs derived from them significantly enhanced the TSC capability which was reflected by COLI and COLIII levels. These results support the potential of EUG-BMSCEV-treated TSCs to regulate tendon matrix formation. Immunohistochemical staining also showed higher expression of both collagen in the EUG-BMSC-EV-treated TSC group than in other groups (Figure 10(c)). After 2 weeks, we can find excellent collagen accumulation in the EUGBMSC-EV group. The expression of COLI was also higher than COLIII, which revealed high quality of tendon healing (Figures 11(a)-11(c)).

\section{Discussion}

TSCs implanted into tendon injury sites are exposed to various cellular stresses that impair their self-renewal, proliferation, and differentiation capabilities [12, 13,31]. Oxidative stress in tendon injury sites has been identified as a major factor that contributes to degenerative events and extracellular matrix organization in tendons [32,33]. Moreover, oxidative stress-induced damage of TSCs provides a potential therapeutic target for tendon repair $[10,12]$. Previous studies have indicated that conditioned medium from MSCs alleviates oxidative stress of expanded umbilical cord blood cells [34]. Recent studies have demonstrated that BMSCs mediate wound healing through secretion of EVs [35-37]. MSC-EVs also suppress liver injury development via antioxidant activities [38]. BMSC-EVs contain various prosurvival factors that reduce apoptosis, inflammation, and oxidative stress during tissue repair and degenerative diseases. Recent studies have also demonstrated the importance of MSC-EVs in regulating the properties of cells during tissue regeneration $[16,39]$. On the basis of these findings, we determined whether BMSC-EVs modulated the impaired function of TSCs under oxidative stress.

In recent years, attention has been focused on the preconditioning of MSCs to enhance their functions. Salidroside reduces the production of ROS in endothelial cells 

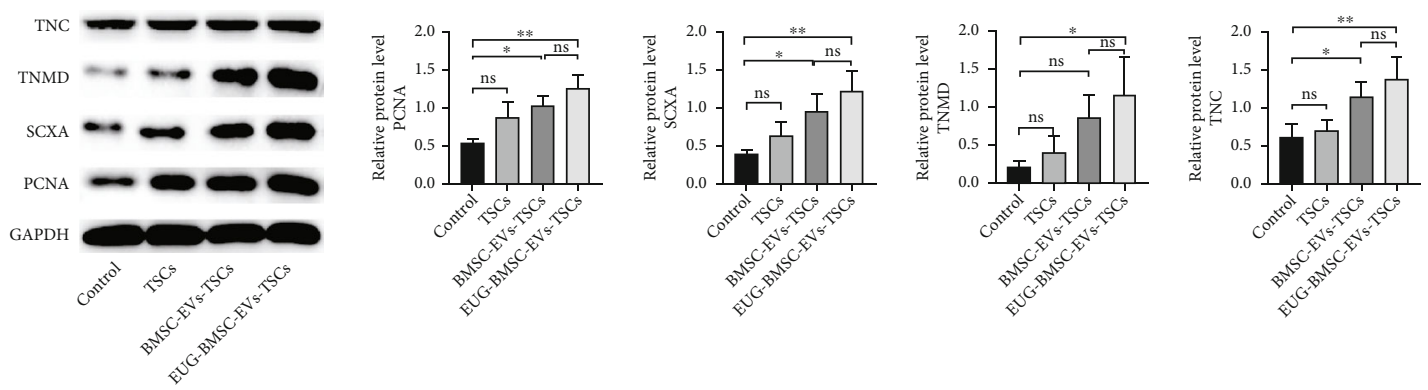

(a)
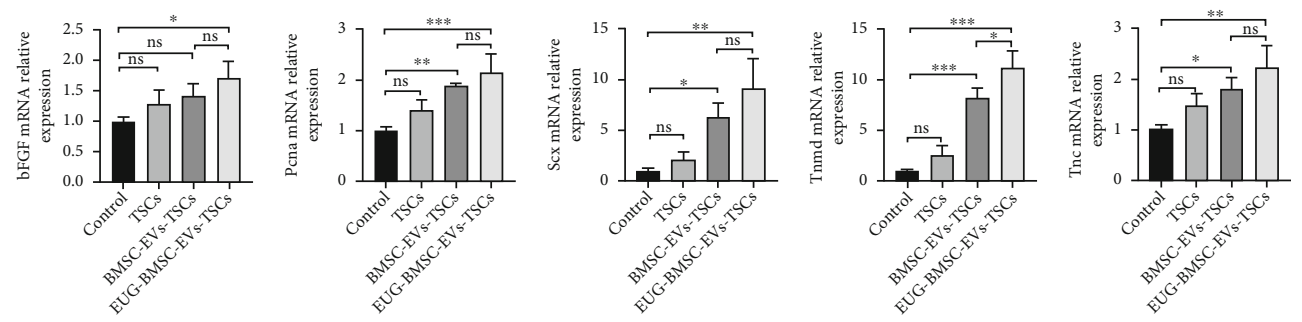

(b)
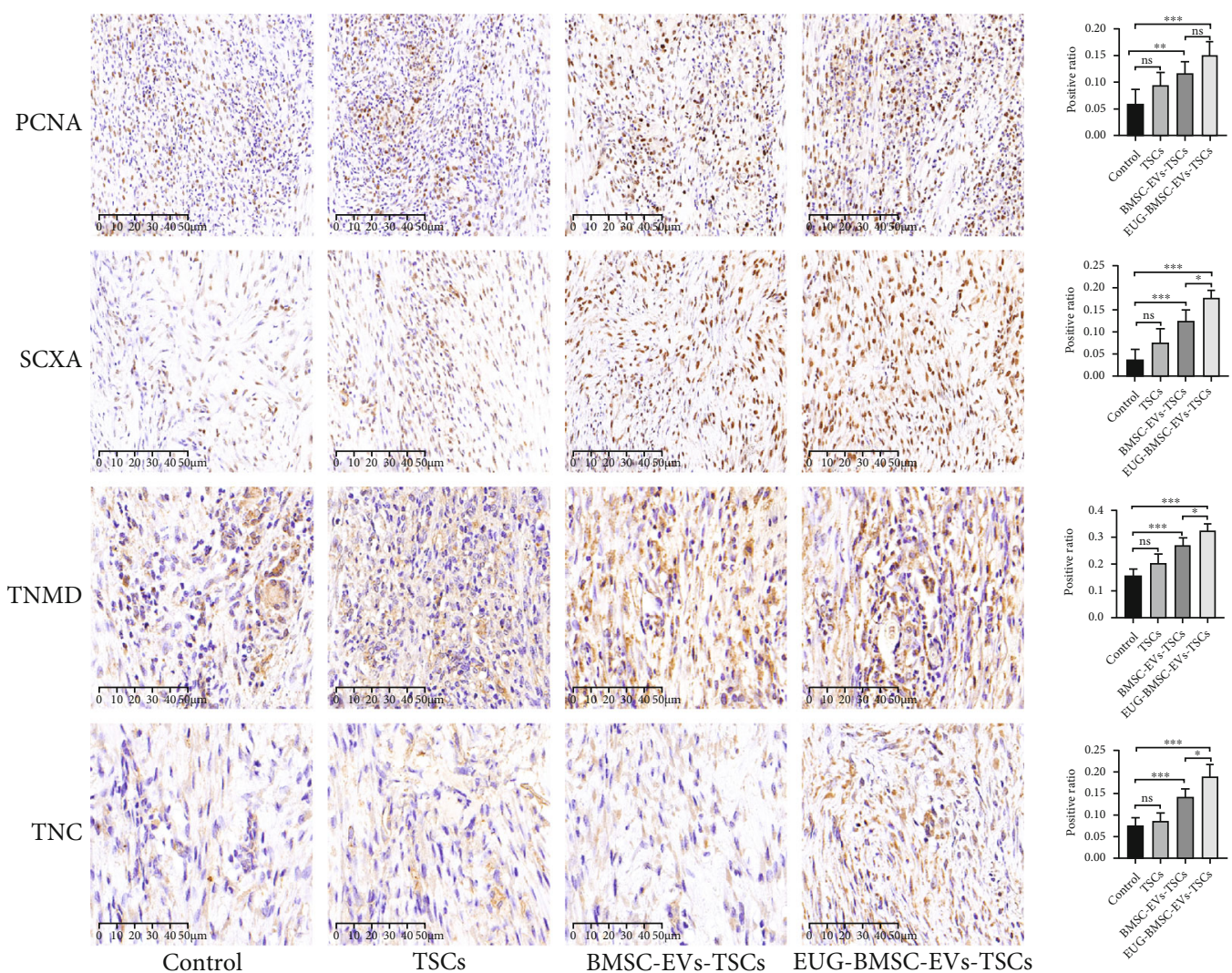

(c)

FigURE 8: EUG-BMSC-EV-pretreated TSCs promoted proliferation and tenogenesis at week 1. (a) Western blot was used to examine the expression of PCNA, SCXA, TNMD, and TNC in tendon injury. (b) The gene expression of bFGF, PCNA, SCX, TNMD, and TNC in tendon injury. (c) Immunohistochemistry assay was performed to assess the expression of PCNA, SCXA, TNMD, and TNC in tendon injury. Bars (PCNA, SCXA): $100 \mu \mathrm{m}$; bars (TNMD, TNC): $50 \mu \mathrm{m}$. Data are represented as mean \pm SD. ${ }^{*} P<0.05,{ }^{* *} P<0.01$, and ${ }^{* * *} P<$ 0.001 . 

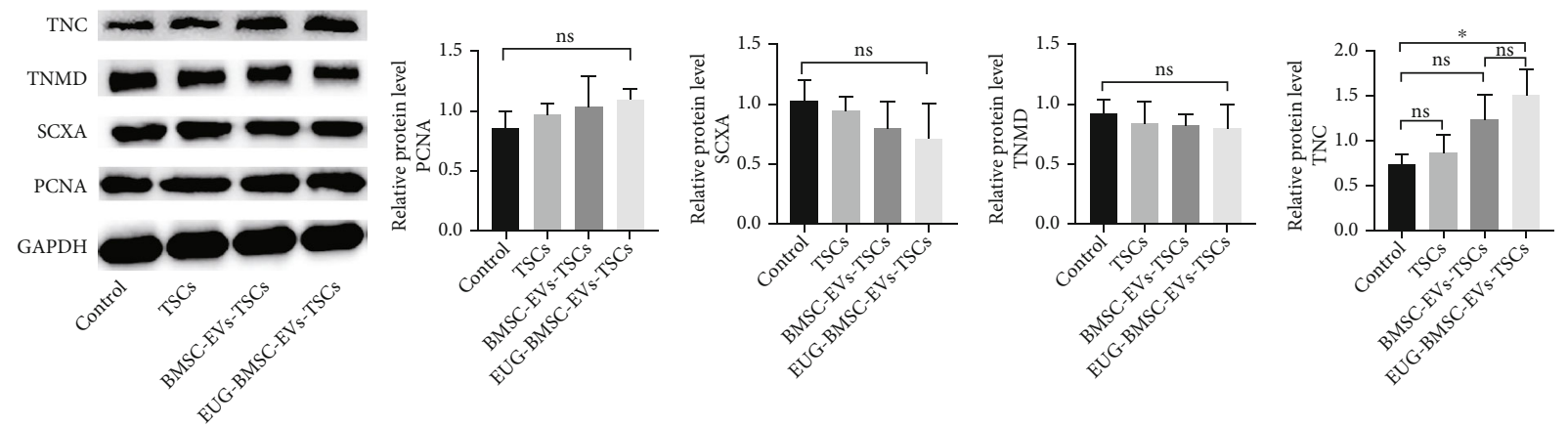

(a)
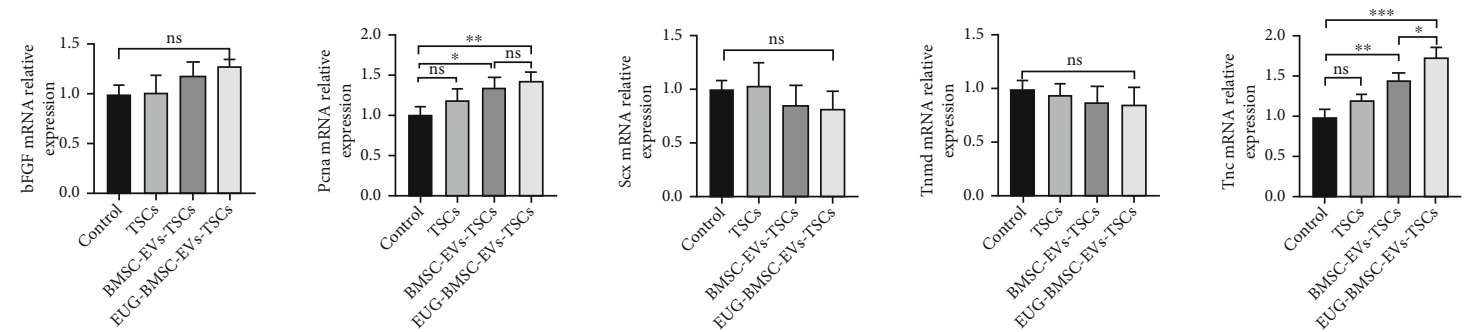

(b)
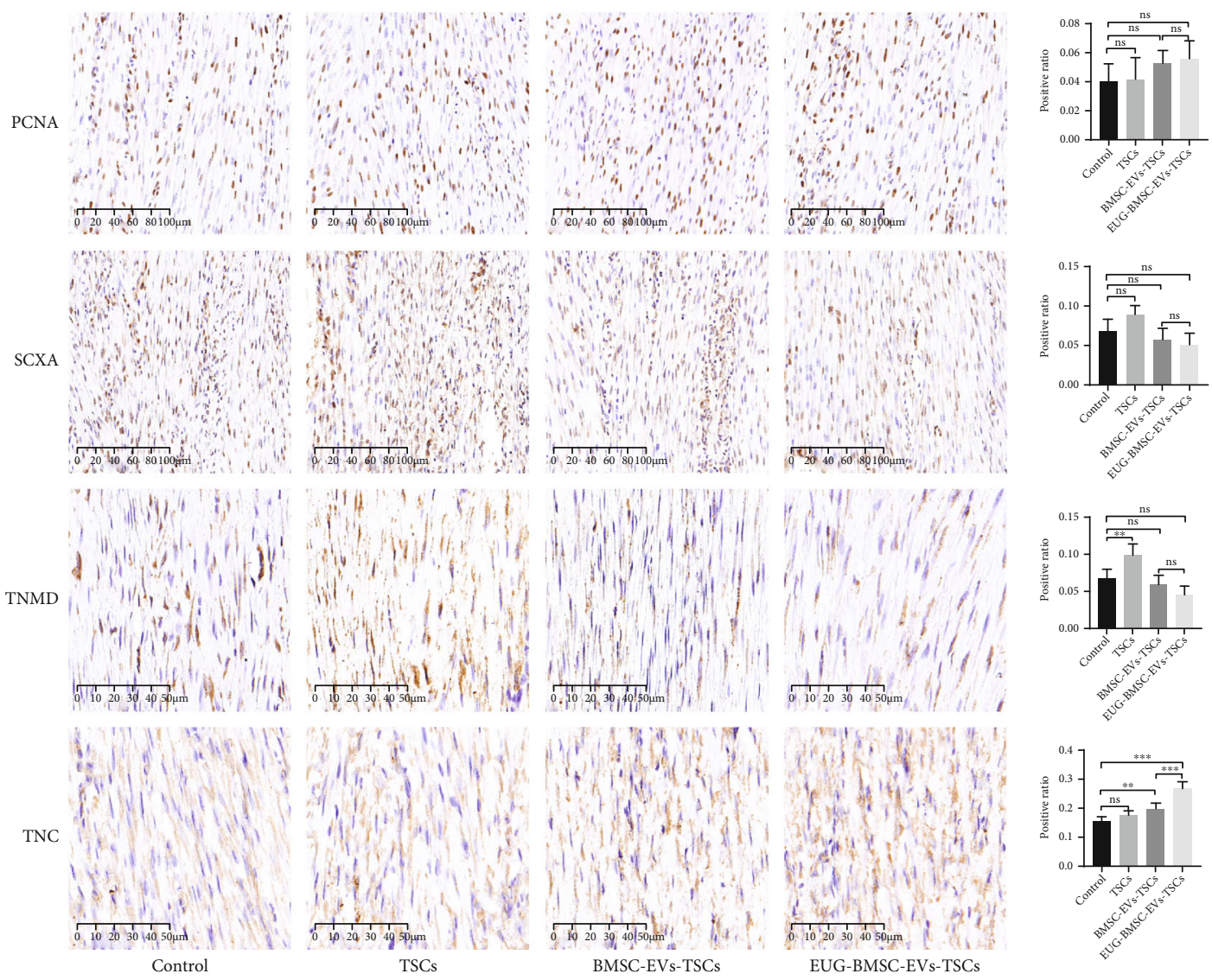

(c)

FIgURE 9: Effects of EUG-BMSC-EV-pretreated TSCs on proliferation and tenogenesis at week 2. (a) Western blot was used to examine the expression of PCNA, SCXA, TNMD, and TNC in tendon injury. (b) The gene expression of bFGF, PCNA, SCX, TNMD, and TNC in tendon injury. (c) Immunohistochemistry assay was performed to assess the expression of PCNA, SCXA, TNMD, and TNC in tendon injury. Bars (PCNA, SCXA): $100 \mu \mathrm{m}$; bars (TNMD, TNC): $50 \mu \mathrm{m}$. Data are represented as mean \pm SD. ${ }^{*} P<0.05,{ }^{* *} P<0.01$, and ${ }^{* * *} P<0.001$. 

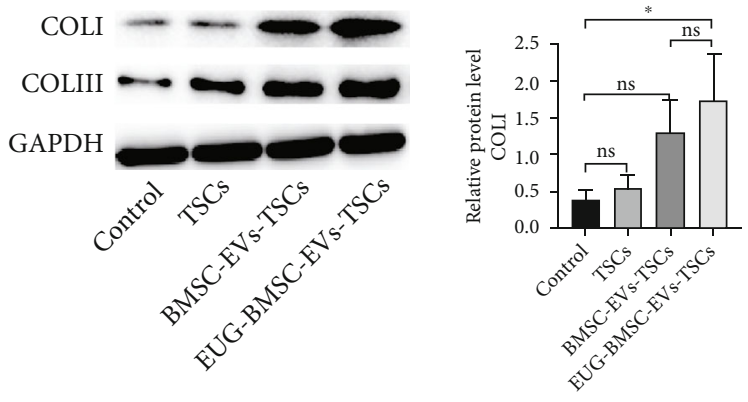

(a)

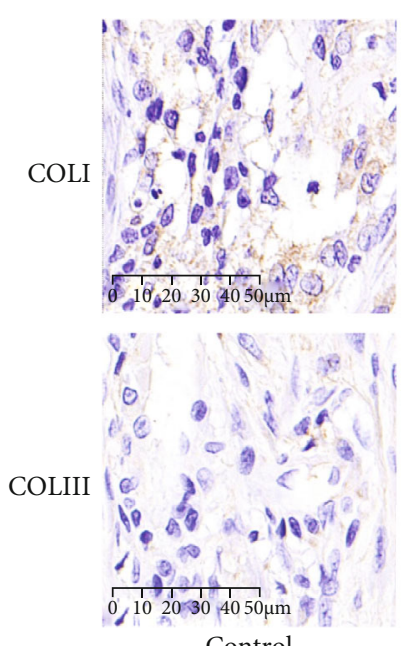

Control

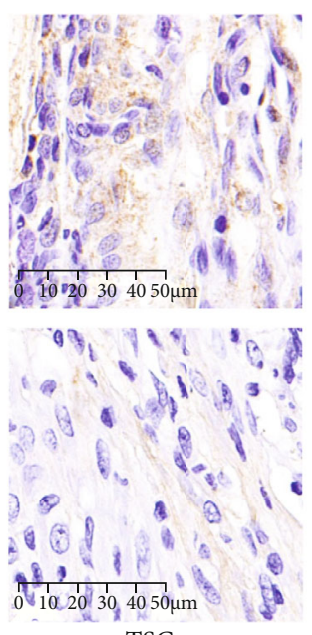

TSCs
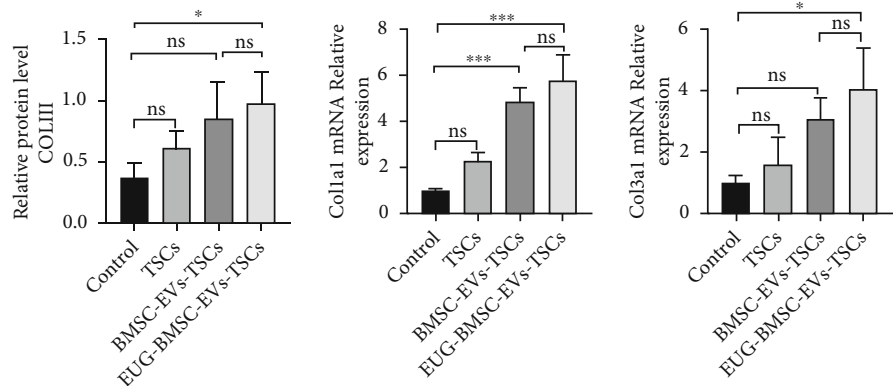

(b)

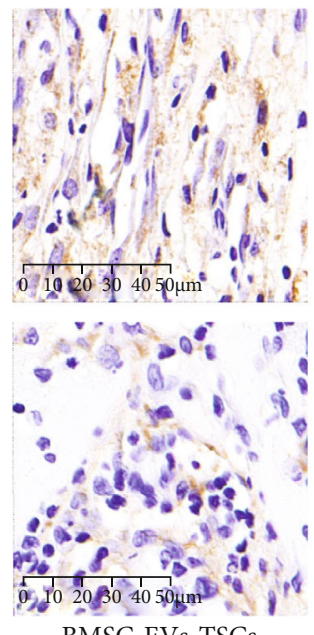

BMSC-EVs-TSCs
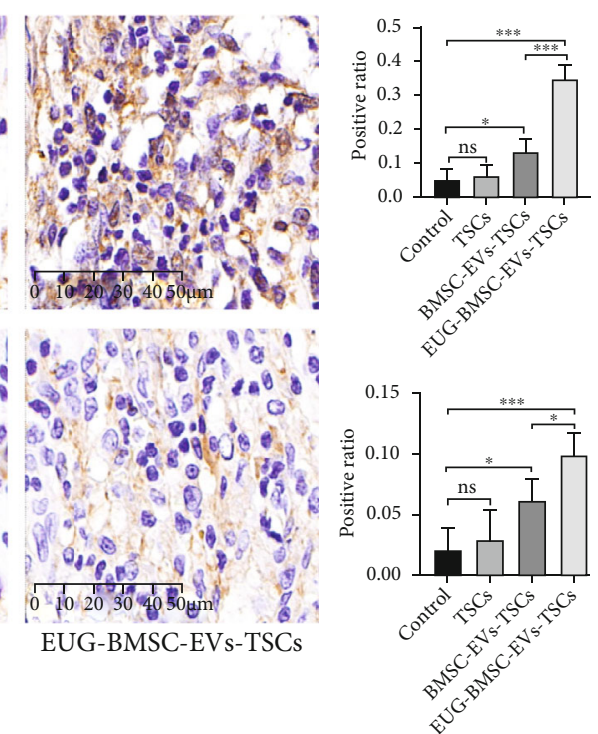

(c)

FIGURE 10: EUG-BMSC-EV-pretreated TSCs enhanced matrix regeneration during tendon healing at week 1. (a) Western blot was used to examine the expression of COLI and COLIII in tendon injury. (b) The expression of Collal and Col3al genes in tendon injury. (c) The expression of COLI and COLIII was detected by immunohistochemistry assay in tendon injury. Bars: $50 \mu \mathrm{m}$. Data are represented as mean \pm SD. ${ }^{*} P<0.05$ and ${ }^{* * *} P<0.001$.

induced by hyperglycemia, thereby regulating apoptosis induced by oxidative stress to play a cellular protective role. Moreover, preconditioning with salidroside enhances the repair function of MSCs in diabetic patients [40]. Preconditioning MSCs with eicosapentaenoic acid also demonstrated superior effects on inflammation as well as tissue remodeling compared to nonpreconditioned MSCs in both models of allergic asthma and sepsis [41, 42]. Hydroxycamptothecin (HCPT), a DNA topoisomerase I inhibitor, inhibits excessive proliferation and induces fibroblast apoptosis. It has been reported that EVs from human bone marrow mesenchymal stem cells pretreated with HCPT have a strong therapeutic effect on tendon adhesion after tendon injury [43]. EVs derived from LPSpreconditioned MSCs better regulate polarization of macrophages and inhibit chronic inflammation [44]. Therefore, we speculated that preconditioned MSCs would have a better therapeutic effect under treatment with certain substances. EUG is a natural anti-inflammatory agent and antioxidant [45]. We hypothesized that EVs derived from EUG-preconditioned BMSCs would have a better antioxidant capacity and would better ameliorate impair- ment of TSCs induced by oxidative stress than BMSCEVs alone.

Cell proliferation and viability are important for tenocyte regeneration. The proliferation of TSCs was decreased by $\mathrm{H}_{2} \mathrm{O}_{2}$, which might account for the poor repair in tendons under oxidative stress. Moreover, we found that $\mathrm{H}_{2} \mathrm{O}_{2}$ significantly impaired the viability of TSCs. These results support that oxidative stress is detrimental to TSCs for tendon healing.

We also investigated the effects of $\mathrm{H}_{2} \mathrm{O}_{2}$ on the tenogenic differentiation potential. TSCs in the $\mathrm{H}_{2} \mathrm{O}_{2}$-treated group expressed significantly lower levels of COLI, TNC, TNMD, and SCX compared with the control group. TNMD is important for early tenogenic differentiation, and SCX positively regulates the expression of TNMD and COLI $[46,47]$. TNC is a glycoprotein of the extracellular matrix, which is fundamental for cell-cell and cell-matrix interactions [48]. Our results showed that oxidative stress impaired the tenogenic differentiation ability of TSCs, which may be a challenge in TSC-based therapy [10]. However, TSCs pretreated with EUG-BMSC-EVs retained their ability, such as proliferation, viability, and tenogenic differentiation. 

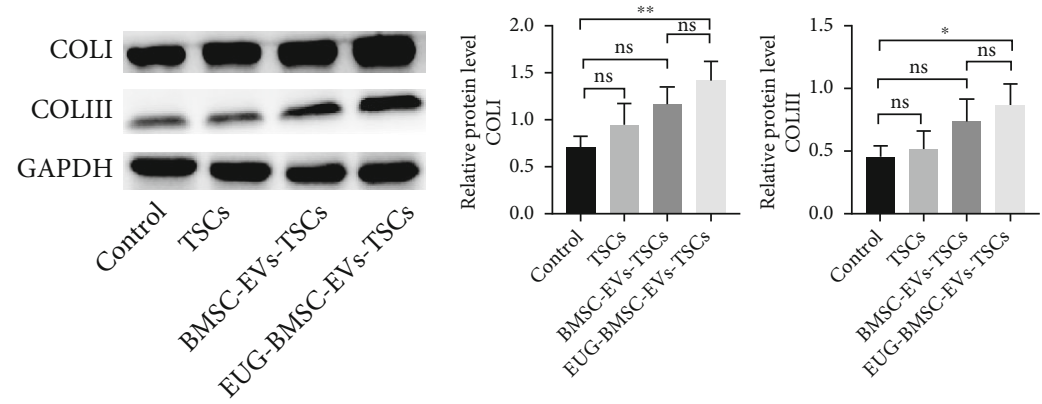

(a)
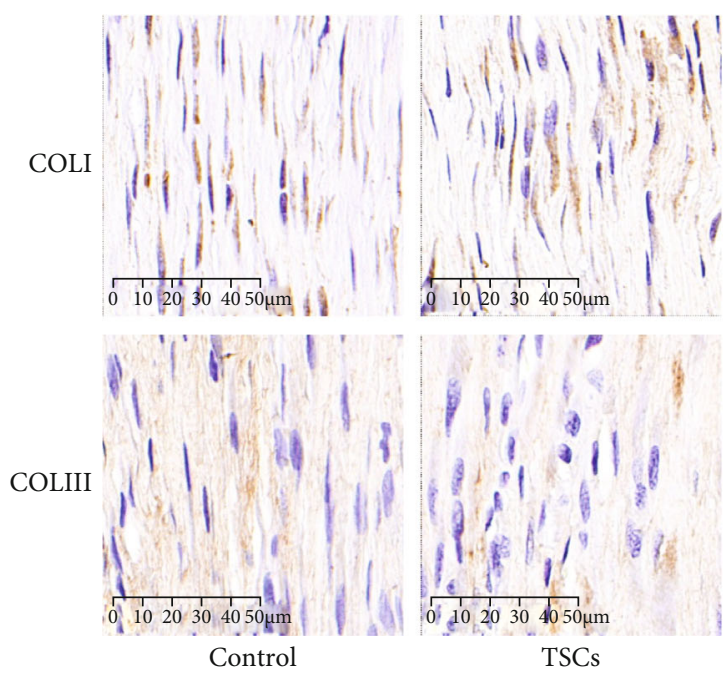

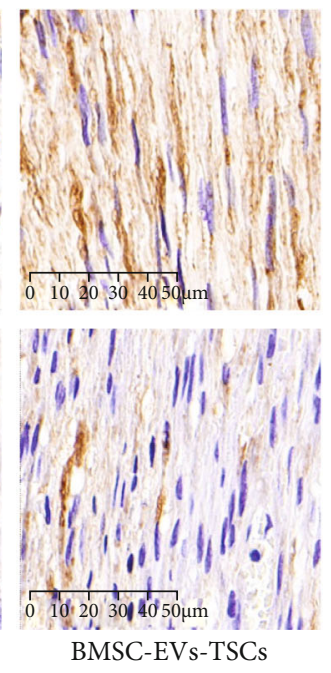

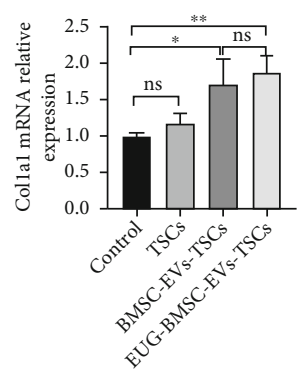

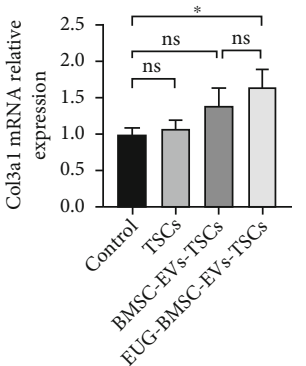

(b)
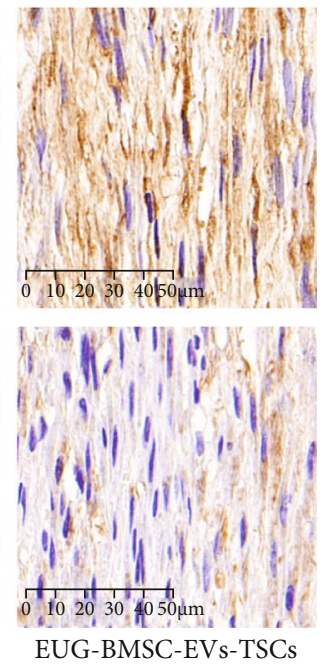
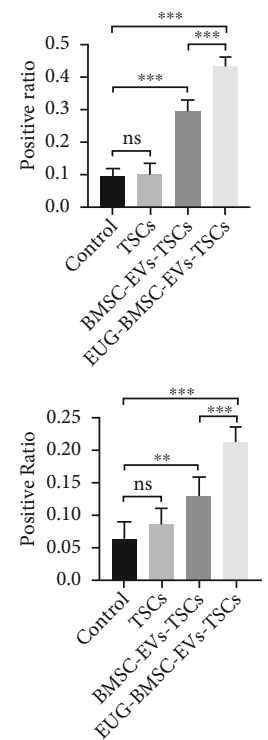

(c)

FigURE 11: Effect of EUG-BMSC-EV-pretreated TSCs on matrix regeneration during tendon healing at week 2. (a) Western blot was used to examine the expression of COLI and COLIII in tendon injury. (b) The expression of Collal and Col3al genes in tendon injury. (c) The expression of COLI and COLIII was detected by immunohistochemistry assay in tendon injury. Bars: $50 \mu \mathrm{m}$. Data are represented as mean \pm SD. ${ }^{*} P<0.05,{ }^{* *} P<0.01$, and ${ }^{* * *} P<0.001$.

Previous reports indicate that oxidative stress is a potential proapoptotic factor in stem cells [49-51]. We found that TSCs exposed to $\mathrm{H}_{2} \mathrm{O}_{2}$ exhibited a significant increase in apoptosis. Increased apoptotic activity in tendon tissue often correlates with an altered tendon tissue composition and damaged matrix during repair. A previous study has shown that BMSC-EVs modulate several biological processes that include cell apoptosis [52]. We speculated that BMSC-EVs may also affect $\mathrm{H}_{2} \mathrm{O}_{2}$-induced apoptosis of TSCs. PARP1 plays an important role in DNA damage repair and is used as an indicator of cell apoptosis. We used PARP1 to reflect TSCs apoptosis [53]. In this study, BMSC-EVs relieved apoptosis of TSCs exposed to oxidative stress. These data also indicated that EUG-BMSC-EVs were superior to BMSCEVs in protecting TSCs from $\mathrm{H}_{2} \mathrm{O}_{2}$-induced damage.

Intracellular oxidative stress decreases collagen expression in tissues, which leads to degeneration [54]. We found that $\mathrm{H}_{2} \mathrm{O}_{2}$-treated TSCs exhibited an increase in ROS accumulation. ROS are essential modulators that control physiological activities during tissue repair, such as cell growth, proliferation, migration, differentiation, and apoptosis. Pro- duction of ROS is involved in the pathological mechanism of tendon degeneration. Catalase, an important antioxidant enzyme in scavenging ROS, plays an important role in maintaining the balance of redox state. In our study, catalase level increased after $\mathrm{H}_{2} \mathrm{O}_{2}$ treatment, which suggested increased oxidative stress and the involvement of ROS. The oxidative stress-induced activation of the Nrf2/HO- 1 pathway results in increased expression of downstream antioxidant enzymes including catalase. The phenomenon was in accordance with a previous study [55]. Considering that BMSC-EVs often function as antioxidants, we detected ROS and catalase levels in TSCs under oxidative stress. As we predicted, treatment with EUG-BMSC-EVs significantly decreased intracellular ROS levels and increased catalase levels in $\mathrm{H}_{2} \mathrm{O}_{2}$-treated TSCs. These results suggested that EUG-BMSC-EVs protected TSCs from oxidative stress.

Nrf2 plays a critical role in preventing cell and tissue damage caused by oxidative stress. It controls the transcription of several cytoprotective genes in response to oxidative stress $[56,57]$. Previous studies have revealed that oxidative stress contributes to tendon degeneration and $\mathrm{Nrf} 2 / \mathrm{HO}-1$ 
upregulation increases the capability of TSCs to cope with $\mathrm{H}_{2} \mathrm{O}_{2}$-induced damage [13]. Our results were consistent with previous findings and showed that cell pretreatment with ML385 (an inhibitor of Nrf2) attenuated EUG-BMSCEV-induced cytoprotective effects. EUG-BMSC-EVs potentially protected against oxidative-related tendon physiological dysfunctions by activating the $\mathrm{Nrf} 2 / \mathrm{HO}-1$ pathway.

Our results also showed that transplantation of TSCs pretreated with EUG-BMSC-EVs promoted tendon repair at 14 days after injury. This was evidenced by the improved fiber alignment score after the transplantation of pretreated TSCs. However, the group implanted with TSCs alone did not show obvious effects. In previous studies, some people found that TSCs were effective, while others found that TSCs alone had little effect $[58,59]$. We found that the TSC group had a better effect than the control group while there was no significant difference in many indexes. We inferred that this result may be associated with the survival of TSCs. Untreated TSCs exhibit limited survival in wound tissue and cause unsatisfactory repair [10]. Pretreatment with EUG-BMSC-EVs enhanced the capability of TSCs to adapt to pathophysiological conditions. The improved tissue quality with EUG-BMSC-EV-treated TSCs was further supported by Masson's trichrome staining results. In particular, more regenerated collagen fibers were observed in the EUG-BMSC-EV-treated TSC group.

In this study, EUG-BMSC-EVs promoted tenogenic differentiation and increased the expression of tendon-related genes and proteins in the healing tendon. Collagen is the major protein in tendons and provides an environment to maintain a stretch for tendon cells [60]. In the patellar tendon injury model, implantation of EUG-BMSC-EV-treated TSCs significantly enhanced COLI and COLIII production.

We also examined the expression of tenocyte-related genes. As expected, the expression of SCX was elevated by EUG-BMSC-EV-pretreated TSCs in healing tendons at 1 week in our study. TNMD is involved in tendon maturation [30], and it was significantly increased in the EUG-BMSCEV-pretreated TSC implantation group. bFGF is involved in fibroblast/tenocyte growth, and its expression was increased in healing tendon tissues following implantation of EUG-BMSC-EV-pretreated TSCs. These findings suggest that EUG-BMSC-EVs maintained the spontaneous tenogenic differentiation and growth potential of TSCs in healing tissues. We also found that these indexes were decreased after 2 weeks. We believe this phenomenon may be correlated with the better repair in the EUG-BMSC-EV group. During the early stage of repair, TSCs pretreated with EUG-BMSC-EVs actively participated in the reconstruction of tendons and accelerated wound tissue recovery. Because this also resulted in rapid consumption of TSCs, the repair process slowed after 2 weeks.

The current study has some limitations. First, we did not explore how EUG enhances the antioxidant capacity of BMSC-EVs. Therefore, the components in EVs altered by EUG remain to be explored. Second, we did not monitor the survival of TSCs in the injured tendons of our animal experiments, and only wound healing and the expression of related proteins in the tendons were used to assess repair.
The survival and function of cells at the tendon also remain to be explored. We will do further research to explore the mechanism and make EUG-BMSC-EVs justifiable for clinical therapy.

\section{Conclusions}

In brief, we demonstrate that EUG-BMSC-EVs could protect TSCs from damage induced by oxidative stress through activating the Nrf2/HO-1 pathway. Implantation of EUGBMSC-EV-pretreated TSCs is a viable approach to improve the quality of tendon healing. These findings provide us with a novel therapeutic strategy for tendon repair in the future.

$\begin{array}{ll}\text { Abbreviations } \\ \text { BCA: } & \text { Bicinchoninic acid } \\ \text { BMSC: } & \text { Bone marrow mesenchymal stem cells } \\ \text { Cat: } & \text { Catalogue } \\ \text { COL: } & \text { Collagen } \\ \text { DMEM: } & \text { Dulbecco's modified Eagle's medium } \\ \text { EUG: } & \text { Eugenol } \\ \text { FBS: } & \text { Fetal bovine serum } \\ \text { GAPDH: } & \text { Glyceraldehyde-3-phosphate dehydrogenase } \\ \text { H\&E: } & \text { Hematoxylin-eosin } \\ \text { HO-1: } & \text { Heme oxygenase 1 } \\ \text { MSCs: } & \text { Mesenchymal stem cells } \\ \text { NTA: } & \text { Nanoparticle tracking analysis } \\ \text { Nrf2/Nfe2l2: } & \text { Nuclear factor, erythroid 2-like 2 } \\ \text { PARP1: } & \text { Poly ADP-ribose polymerase 1 } \\ \text { PBS: } & \text { Phosphate-buffered saline } \\ \text { PCNA: } & \text { Proliferating cell nuclear antigen } \\ \text { ROS: } & \text { Reactive oxygen species } \\ \text { SCX: } & \text { Scleraxis } \\ \text { SD: } & \text { Standard deviation } \\ \text { TEM: } & \text { Transmission electron microscopy } \\ \text { TNMD: } & \text { Tenomodulin } \\ \text { TNC: } & \text { Tenascin C } \\ \text { TSCs: } & \text { Tendon stem/progenitor cells } \\ & \end{array}$

\section{Data Availability}

All data generated or analyzed during this study are included in this manuscript.

\section{Ethical Approval}

The study was approved by the Ethics Committee of Shanghai Children's Medical Center, Shanghai Jiao Tong University School of Medicine (China).

\section{Conflicts of Interest}

The authors declare that they have no competing interests.

\section{Authors' Contributions}

XZL and ZS carried out the main part of the studies and drafted the manuscript. KYS, QW, CCX, FQW, and YCZ analyzed the results. KYS and QW reviewed the manuscript. 
DPJ participated in the design of the study and performed the statistical analysis. All authors read and approved the final manuscript. XZL and ZS contributed equally to this work.

\section{Acknowledgments}

We thank Melissa Crawford, Ph.D., and Mitchell Arico, from Liwen Bianji (Edanz) (http://www.liwenbianji.cn/), for editing the English text of a draft of this manuscript. This project was supported by Grants from the National Natural Science Foundation of China (grant numbers 81972139).

\section{References}

[1] D. S. Pillai, B. S. Dhinsa, and W. S. Khan, "Tissue engineering in Achilles tendon reconstruction; the role of stem cells, growth factors and scaffolds," Current Stem Cell Research \& Therapy, vol. 12, no. 6, pp. 506-512, 2017.

[2] W. Morita, S. G. Dakin, S. J. B. Snelling, and A. J. Carr, "Cytokines in tendon disease," Bone \& Joint Research, vol. 6, no. 12, pp. 656-664, 2017.

[3] A. J. Turlo, Y. Ashraf Kharaz, P. D. Clegg, J. Anderson, and M. J. Peffers, "Donor age affects proteome composition of tenocyte-derived engineered tendon," BMC Biotechnology, vol. 18, no. 1, p. 2, 2018.

[4] H. Shen, R. Jayaram, S. Yoneda et al., "The effect of adiposederived stem cell sheets and CTGF on early flexor tendon healing in a canine model," Scientific Reports, vol. 8, no. 1, article 11078, 2018.

[5] Y. Zhou, J. Zhang, H. Wu, M. V. Hogan, and J. H. C. Wang, "The differential effects of leukocyte-containing and pure platelet-rich plasma (PRP) on tendon stem/progenitor cells implications of PRP application for the clinical treatment of tendon injuries," Stem Cell Research \& Therapy, vol. 6, no. 1, p. 173,2015

[6] B. Blum, O. Bar-Nur, T. Golan-Lev, and N. Benvenisty, “The anti-apoptotic gene survivin contributes to teratoma formation by human embryonic stem cells," Nature Biotechnology, vol. 27, no. 3, pp. 281-287, 2009.

[7] C. Zhang, E. Zhang, L. Yang et al., "Histone deacetylase inhibitor treated cell sheet from mouse tendon stem/progenitor cells promotes tendon repair," Biomaterials, vol. 172, pp. 66-82, 2018.

[8] S. Tarafder, E. Chen, Y. Jun et al., “Tendon stem/progenitor cells regulate inflammation in tendon healingviaJNK and STAT3 signaling," FASEB Journal, vol. 31, no. 9, pp. 39913998, 2017.

[9] J. Chen, E. Zhang, W. Zhang et al., "FosPromotes early stage teno-lineage differentiation of tendon stem/progenitor cells in tendon," Stem Cells Translational Medicine, vol. 6, no. 11, pp. 2009-2019, 2017.

[10] Y. W. Lee, S. C. Fu, M. Y. Yeung, C. M. Lau, K. M. Chan, and L. K. Hung, "Effects of redox modulation on cell proliferation, viability, and migration in cultured rat and human tendon progenitor cells," Oxidative Medicine and Cellular Longevity, vol. 2017, Article ID 8785042, 8 pages, 2017.

[11] C. Tang, Y. Chen, J. Huang et al., "The roles of inflammatory mediators and immunocytes in tendinopathy," Journal of Orthopaedic Translation, vol. 14, pp. 23-33, 2018.
[12] H. Chen, H. A. Ge, G. B. Wu, B. Cheng, Y. Lu, and C. Jiang, "Autophagy prevents oxidative stress-induced loss of selfrenewal capacity and stemness in human tendon stem cells by reducing ROS accumulation," Cellular Physiology and Biochemistry, vol. 39, no. 6, pp. 2227-2238, 2016.

[13] W. Sun, J. Meng, Z. Wang et al., "Proanthocyanidins attenuation of HO-induced oxidative damage in tendon-derived stem cells via upregulating Nrf-2 signaling pathway," BioMed Research International, vol. 2017, Article ID 7529104, 8 pages, 2017.

[14] L. K. Hung, S. C. Fu, Y. W. Lee, T. Y. Mok, and K. M. Chan, "Local vitamin-C injection reduced tendon adhesion in a chicken model of flexor digitorum profundus tendon injury," Journal of Bone and Joint Surgery, vol. 95, no. 7, article e41, 2013.

[15] W. Liu, Y. Wang, F. Gong et al., "Exosomes derived from bone mesenchymal stem cells repair traumatic spinal cord injury by suppressing the activation of A1 neurotoxic reactive astrocytes," Journal of Neurotrauma, vol. 36, no. 3, pp. 469-484, 2019.

[16] S. C. Abreu, M. Lopes-Pacheco, D. J. Weiss, and P. R. M. Rocco, "Mesenchymal stromal cell-derived extracellular vesicles in lung diseases: current status and perspectives," Frontiers in Cell and Developmental Biology, vol. 9, article 600711, 2021.

[17] J. Yang, X. X. Liu, H. Fan et al., "Extracellular vesicles derived from bone marrow mesenchymal stem cells protect against experimental colitis via attenuating colon inflammation, oxidative stress and apoptosis," PLoS One, vol. 10, no. 10, article e0140551, 2015.

[18] S. Firoozi, S. Pahlavan, M. H. Ghanian et al., "Mesenchymal stem cell-derived extracellular vesicles alone or in conjunction with a SDKP-conjugated self-assembling peptide improve a rat model of myocardial infarction," Biochemical and Biophysical Research Communications, vol. 524, no. 4, pp. 903-909, 2020.

[19] Y. Bai, Y. D. Han, X. L. Yan et al., “Adipose mesenchymal stem cell-derived exosomes stimulated by hydrogen peroxide enhanced skin flap recovery in ischemia-reperfusion injury," Biochemical and Biophysical Research Communications, vol. 500, no. 2, pp. 310-317, 2018.

[20] Z. Shi, Q. Wang, and D. Jiang, "Extracellular vesicles from bone marrow-derived multipotent mesenchymal stromal cells regulate inflammation and enhance tendon healing," Journal of Translational Medicine, vol. 17, no. 1, p. 211, 2019.

[21] M. Bai, L. Zhang, B. Fu et al., "IL-17A improves the efficacy of mesenchymal stem cells in ischemic-reperfusion renal injury by increasing Treg percentages by the COX-2/PGE2 pathway," Kidney International, vol. 93, no. 4, pp. 814-825, 2018.

[22] Y. Liu, H. Ren, Y. Zhou et al., “The hypoxia conditioned mesenchymal stem cells promote hepatocellular carcinoma progression through YAP mediated lipogenesis reprogramming," Journal of Experimental \& Clinical Cancer Research, vol. 38, no. 1, p. 228, 2019.

[23] Z. Yang, C. He, J. He, J. Chu, H. Liu, and X. Deng, "Curcuminmediated bone marrow mesenchymal stem cell sheets create a favorable immune microenvironment for adult full-thickness cutaneous wound healing," Stem Cell Research \& Therapy, vol. 9, no. 1, p. 21, 2018.

[24] Y. Hu, R. Tao, L. Chen et al., "Exosomes derived from pioglitazone-pretreated MSCs accelerate diabetic wound healing through enhancing angiogenesis," Journal of Nanobiotechnology, vol. 19, no. 1, p. 150, 2021. 
[25] M. Ulanowska and B. Olas, "Biological properties and prospects for the application of eugenol-a review," International Journal of Molecular Sciences, vol. 22, no. 7, p. 3671, 2021.

[26] M. Fathy, M. Okabe, H. M. Saad Eldien, and T. Yoshida, “ATMSCs antifibrotic activity is improved by eugenol through modulation of TGF- $\beta /$ Smad signaling pathway in rats," Molecules, vol. 25, no. 2, p. 348, 2020.

[27] L. L. Wang, X. F. Yin, X. C. Chu, Y. B. Zhang, and X. N. Gong, "Retracted: Platelet-derived growth factor subunit B is required for tendon-bone healing using bone marrowderived mesenchymal stem cells after rotator cuff repair in rats," Journal of Cellular Biochemistry, vol. 119, no. 11, pp. 8897-8908, 2018.

[28] Y. Bi, D. Ehirchiou, T. M. Kilts et al., "Identification of tendon stem/progenitor cells and the role of the extracellular matrix in their niche," Nature Medicine, vol. 13, no. 10, pp. 1219-1227, 2007.

[29] Y. Sun, H. Chen, H. Ye et al., "Nudt21-mediated alternative polyadenylation of HMGA2 3 '-UTR impairs stemness of human tendon stem cell," Aging, vol. 12, no. 18, pp. 1843618452,2020

[30] D. Lin, P. Alberton, M. D. Caceres, E. Volkmer, M. Schieker, and D. Docheva, "Tenomodulin is essential for prevention of adipocyte accumulation and fibrovascular scar formation during early tendon healing," Cell Death \& Disease, vol. 8, no. 10, article e3116, 2017.

[31] D. Jiang, B. Xu, M. Yang, Z. Zhao, Y. Zhang, and Z. Li, "Efficacy of tendon stem cells in fibroblast-derived matrix for tendon tissue engineering," Cytotherapy, vol. 16, no. 5, pp. 662673, 2014.

[32] N. N. DePhillipo, Z. S. Aman, M. I. Kennedy, J. P. Begley, G. Moatshe, and R. F. LaPrade, "Efficacy of vitamin C supplementation on collagen synthesis and oxidative stress after musculoskeletal injuries: a systematic review," Orthopaedic Journal of Sports Medicine, vol. 6, no. 10, 2018.

[33] R. J. Kim, Y. S. Hah, C. M. Sung, J. R. Kang, and H. B. Park, "Do antioxidants inhibit oxidative-stress-induced autophagy of tenofibroblasts?," Journal of Orthopaedic Research, vol. 32, no. 7, pp. 937-943, 2014.

[34] D. Kadekar, S. Rangole, V. Kale, and L. Limaye, "Conditioned medium from placental mesenchymal stem cells reduces oxidative stress during the cryopreservation of ex vivo expanded umbilical cord blood cells," PLoS One, vol. 11, no. 10, article e0165466, 2016.

[35] P. Wu, B. Zhang, H. Shi, H. Qian, and W. Xu, "MSC-exosome: a novel cell-free therapy for cutaneous regeneration," Cytotherapy, vol. 20, no. 3, pp. 291-301, 2018.

[36] Q. Shi, Z. Qian, D. Liu et al., "GMSC-derived exosomes combined with a chitosan/silk hydrogel sponge accelerates wound healing in a diabetic rat skin defect model," Frontiers in Physiology, vol. 8, p. 904, 2017.

[37] Y. J. Kim, S. M. Yoo, H. H. Park et al., "Exosomes derived from human umbilical cord blood mesenchymal stem cells stimulates rejuvenation of human skin," Biochemical and Biophysical Research Communications, vol. 493, no. 2, pp. 1102-1108, 2017.

[38] W. Jiang, Y. Tan, M. Cai et al., "Human umbilical cord MSCderived exosomes suppress the development of CCl-induced liver injury through antioxidant effect," Stem Cells International, vol. 2018, Article ID 6079642, 11 pages, 2018.

[39] S. Zhang, S. J. Chuah, R. C. Lai, J. H. P. Hui, S. K. Lim, and W. S. Toh, "MSC exosomes mediate cartilage repair by enhancing proliferation, attenuating apoptosis and modulating immune reactivity," Biomaterials, vol. 156, pp. 16-27, 2018.

[40] A. D. Ariyanti, J. Zhang, O. Marcelina et al., "Salidroside-pretreated mesenchymal stem cells enhance diabetic wound healing by promoting paracrine function and survival of mesenchymal stem cells under hyperglycemia," Stem Cells Translational Medicine, vol. 8, no. 4, pp. 404-414, 2019.

[41] J. D. Silva, M. Lopes-Pacheco, L. L. de Castro et al., "Eicosapentaenoic acid potentiates the therapeutic effects of adipose tissue-derived mesenchymal stromal cells on lung and distal organ injury in experimental sepsis," Stem Cell Research \& Therapy, vol. 10, no. 1, p. 264, 2019.

[42] S. C. Abreu, M. Lopes-Pacheco, A. L. da Silva et al., "Eicosapentaenoic acid enhances the effects of mesenchymal stromal cell therapy in experimental allergic asthma," Frontiers Immunolgy, vol. 9, p. 1147, 2018.

[43] J. Li, Z. Yao, H. Xiong et al., "Extracellular vesicles from hydroxycamptothecin primed umbilical cord stem cells enhance anti-adhesion potential for treatment of tendon injury," Stem Cell Research \& Therapy, vol. 11, no. 1, p. 500, 2020.

[44] D. Ti, H. Hao, C. Tong et al., "LPS-preconditioned mesenchymal stromal cells modify macrophage polarization for resolution of chronic inflammation via exosome-shuttled let-7b," Journal of Translational Medicine, vol. 13, no. 1, p. 308, 2015.

[45] J. N. Barboza, C. S. M. B. Filho, R. O. Silva, J. V. R. Medeiros, and D. P. Sousa, "An overview on the anti-inflammatory potential and antioxidant profile of eugenol," Oxidative Medicine and Cellular Longevity, vol. 2018, Article ID 3957262, 9 pages, 2018.

[46] R. Schweitzer, J. H. Chyung, L. C. Murtaugh et al., "Analysis of the tendon cell fate using scleraxis, a specific marker for tendons and ligaments," Development, vol. 128, no. 19, pp. 3855-3866, 2001.

[47] S. Dex, P. Alberton, L. Willkomm et al., "Tenomodulin is required for tendon endurance running and collagen I fibril adaptation to mechanical load," EBioMedicine, vol. 20, pp. 240-254, 2017.

[48] S. P. Roth, S. Schubert, P. Scheibe, C. Groß, W. Brehm, and J. Burk, "Growth factor-mediated tenogenic induction of multipotent mesenchymal stromal cells is altered by the microenvironment of tendon matrix," Cell Transplantation, vol. 27, no. 10, pp. 1434-1450, 2018.

[49] H. Cao, Y. Zhi, H. Xu, H. Fang, and X. Jia, "Zearalenone causes embryotoxicity and induces oxidative stress and apoptosis in differentiated human embryonic stem cells," Toxicology in Vitro, vol. 54, pp. 243-250, 2019.

[50] S. Shin, J. W. Choi, S. Lim et al., "Anti-apoptotic effects of adipose-derived adherent stromal cells in mesenchymal stem cells exposed to oxidative stress," Cell Biochemistry and Function, vol. 36, no. 5, pp. 263-272, 2018.

[51] J. Tian, L. Gu, A. Adams, X. Wang, and R. Huang, "Pellino-1 protects periodontal ligament stem cells against $\mathrm{H} 2 \mathrm{O} 2$ Induced apoptosis via activation of NF- $\kappa \mathrm{B}$ signaling," Molecular Biotechnology, vol. 60, no. 8, pp. 533-538, 2018.

[52] X. Cheng, G. Zhang, L. Zhang et al., "Mesenchymal stem cells deliver exogenous miR-21viaexosomes to inhibit nucleus pulposus cell apoptosis and reduce intervertebral disc degeneration," Journal of Cellular and Molecular Medicine, vol. 22, no. 1, pp. 261-276, 2018.

[53] A. Ray Chaudhuri and A. Nussenzweig, "The multifaceted roles of PARP1 in DNA repair and chromatin remodelling," 
Nature Reviews Molecular Cell Biology, vol. 18, no. 10, pp. 610621, 2017.

[54] A. Galicka, R. Krętowski, J. Nazaruk, and M. CechowskaPasko, "Anethole prevents hydrogen peroxide-induced apoptosis and collagen metabolism alterations in human skin fibroblasts," Molecular and Cellular Biochemistry, vol. 394, no. 1-2, pp. 217-224, 2014.

[55] R. Prakash, E. Fauzia, A. J. Siddiqui et al., "Oxidative stress enhances autophagy-mediated death of stem cells through Erk1/2 signaling pathway - implications for neurotransplantations," Stem Cell Reviews and Reports, vol. 17, no. 6, pp. 23472358, 2021.

[56] B. Huang, J. Liu, S. Fu et al., " $\alpha$-Cyperone attenuates H2O2Induced oxidative stress and apoptosis in SH-SY5Y cells via activation of Nrf2," Frontiers in Pharmacology, vol. 11, p. 281, 2020.

[57] S. H. Aladaileh, O. E. Hussein, M. H. Abukhalil et al., "Formononetin upregulates $\mathrm{Nrf} 2 / \mathrm{HO}-1$ signaling and prevents oxidative stress, inflammation, and kidney injury in methotrexateinduced rats," Antioxidants, vol. 8, no. 10, p. 430, 2019.

[58] I. Komatsu, J. H. C. Wang, K. Iwasaki, T. Shimizu, and T. Okano, "The effect of tendon stem/progenitor cell (TSC) sheet on the early tendon healing in a rat Achilles tendon injury model," Acta Biomaterialia, vol. 42, pp. 136-146, 2016.

[59] L. Chen, J. P. Liu, K. L. Tang et al., “Tendon derived stem cells promote platelet-rich plasma healing in collagenase-induced rat Achilles tendinopathy," Cellular Physiology and Biochemistry, vol. 34, no. 6, pp. 2153-2168, 2014.

[60] L. Gaut and D. Duprez, "Tendon development and diseases," Wiley Interdisciplinary Reviews Developmental Biology, vol. 5, no. 1, pp. 5-23, 2016. 